\title{
Auf den Rangplatz kommt es an - Wirkung der Rangfolge von Produkten auf das Entscheidungsverhalten
}
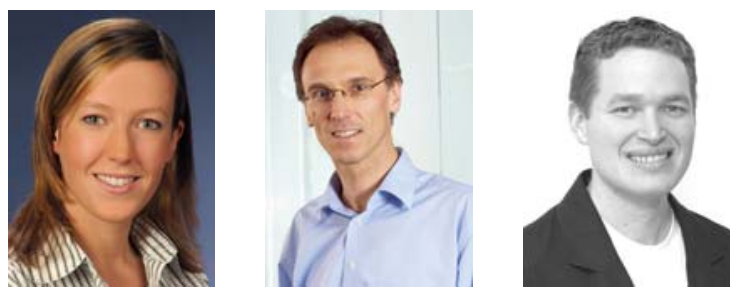

\section{Carina Leesch • Andreas Herrmann • Jan R. Landwehr}

Zusammenfassung: Häufig präsentieren Anbieter ihren Nachfragern die zur Auswahl stehenden Produkte in Listen. Beispiele hierfür bilden Online-Shops (z. B. für Nahrungsmittel, Elektroartikel oder Kleider), die ihre Erzeugnisse zumeist unter Berücksichtigung der vom Kunden gewünschten Auswahlkriterien in einer Rangfolge darbieten. Auch Auktionsportale, Suchmaschinen sowie Wohnungs-, Partner- oder Pkw-Börsen zeigen die für den Kunden als relevant erachteten Erzeugnisse bzw. Treffer in einer Liste. Aus theoretischen Ansätzen, die vor allem aus der deskriptiven Entscheidungstheorie stammen, lässt sich die Vermutung ableiten, dass der Rangplatz eines Produkts in einer Liste einen Effekt auf die Präferenz des Kunden für dieses Gut ausübt. Im Rahmen einer empirischen Studie im Markt für gebrauchte Fahrzeuge kann dieser Zusammenhang bestätigt werden. Bestimmte Rangplätze sind unabhängig von der Beschaffenheit der an diesen Stellen präsentierten Erzeugnisse bei den Individuen beliebter als andere. Für Hersteller und Händler (etwa Online-Shops) liefert die Studie Hinweise über den Zusammenhang zwischen der Art und Weise der Gestaltung von Listen (z. B. geordnet nach einem Kriterium oder randomisiert) und der Neigung von Individuen, sich für das erste, das mittlere etc. Produkt zu entscheiden. Wissenschaftler erlangen ein tieferes Verständnis über individuelles Verhalten bei der Wahl eines Produkts aus einer Liste und der dieses Verhalten determinierenden Faktoren.

Eingegangen: 11.06.2009 / Online publiziert: 04.02.2010

(C) Gabler-Verlag 2009

Dipl.-Soz. Wiss. C. Leesch $(\bowtie)$ · Prof. Dr. A. Herrmann · Dr. J. R. Landwehr

Forschungsstelle für Customer Insight, Universität St. Gallen

Rosenbergstr. 51, 9000 St. Gallen, Schweiz

E-Mail: carina.leesch@unisg.ch

Prof. Dr. A. Herrmann

E-Mail: andreas.herrmann@unisg.ch

Dr. J. R. Landwehr

E-Mail: jan.landwehr@unisg.ch 
Schlüsselwörter: Produktanordnung $\cdot$ Rangordnung $\cdot$ Rangliste $\cdot$ Kompromisseffekt · Marketplace Metacognition · Prospect Theory $\cdot$ Recency und Primacy $\cdot$ experimentelles Design

JEL Classification: M31 - D81

\section{Notwendigkeit einer Analyse der Wirkung der Produktrangfolge auf das Entscheidungsverhalten}

Ein Blick auf das Geschehen in vielen online-Märkten zeigt, dass Unternehmen ihre Erzeugnisse häufig in einer Rangliste ${ }^{1}$ präsentieren. Besonders oft tauchen Listen auf, in denen ein Anbieter seine Produkte zum Verkauf offeriert. Diese Güter sind in der Regel neu und können von beliebig vielen Kunden in beliebiger Anzahl erworben werden. Hierzu zählen Online-Shops für Kleider, Nahrungsmitteln, Kosmetikartikel etc. sowie Angebote von Musik- und Film-Downloads, Buchungsportale für Reisen oder Mass-CustomizationPortale (Piller 2004, S. 316 ff.). Des Weiteren finden sich im Internet Verkaufslisten, in denen verschiedene Anbieter (häufig Privatpersonen) ein einziges Produkt zum Verkauf darbieten. Hierzu gehören etwa Pkw- oder Wohnungsbörsen sowie Auktionsportale; letztere unterscheiden sich jedoch im Hinblick auf das Verkaufsprinzip und die Listensortierung von den bisher genannten Listen. Abschließend sind Suchmaschinen zu erwähnen, die insbesondere der Kontaktanbahnung mit Unternehmen dienen. Selbst in der Offline-Welt sind die zur Auswahl stehenden Optionen zumeist in einer Liste präsentiert. Man denke etwa an Speise- und Getränkekarten in Restaurants, an Werbeprospekte von Supermärkten oder an Verkaufskataloge und -broschüren aller Art. Der Fokus der folgenden Untersuchung richtet sich jedoch auf Verkaufslisten im Internet.

Ein Blick auf die Logik der Anordnung von Produkten in Listen zeigt, dass viele Anbieter einen Zusammenhang zwischen dem Rangplatz, auf dem ein Erzeugnis offeriert ist, und der Präferenz der Nachfrager für dieses Gut (unabhängig von dessen Beschaffenheit) vermuten. Gespräche mit Verantwortlichen von Online-Shops sowie Auktions- und Buchungsportalen liefern den Befund, dass Vorstellungen über das Entscheidungsverhalten der Individuen bei der Gestaltung dieser Systeme Berücksichtigung finden. ${ }^{2}$ Beispielsweise scheinen in vielen Gebrauchtwagenbörsen die aus Unternehmensperspektive attraktiven Fahrzeuge (etwa die mit einem beachtlichen Deckungsbeitrag) auf den ersten Rangplätzen in der den Kunden präsentierten Liste aufzutauchen. Auch Anbieter von Produkt- oder Trefferlisten offerieren Firmen gegen Bezahlung die Option, ihre Erzeugnisse auf den ersten Listenplätzen darzubieten oder beispielsweise farbig hervorzuheben (z. B. ebay.de, hood.de, google.de). Offenbar liegt vielen dieser Systeme die (wohl eher durch Intuition und weniger durch Marktforschung abgeleitete) Idee zugrunde, eine Person interessiere sich vor allem für die zu Anfang auf einer Liste präsentierten oder für die (farblich) hervorgehobenen Optionen.

Obgleich diese Fragestellung in der Literatur bislang kaum Beachtung fand, ${ }^{3}$ bestätigen erste Arbeiten zu diesem Thema die in der Praxis gewonnene Vermutung über die Wirkung der Rangfolge von Produkten auf das Entscheidungsverhalten der Individuen. Bereits in den 60er Jahren konnte Day (1969, S. 99 ff.) nachweisen, dass bei Produkttests, bestehend aus zwei Erzeugnissen, die Probanden zumeist eine Präferenz für das 
zuerst dargebotene Gut entwickelten (Eisenberg u. Barry 1988, S. 295 ff.). Dieser Befund wurde von Dean (1980, S. 109 ff.) repliziert, indem er den Geschmack von Speisen und Getränken durch Probanden beurteilen ließ. In einer Studie von Gierl u. Höser (2002, S. 10 ff.) bewerteten Personen ein Produkt besser, sofern es vor (und nicht nach) einem anderen Erzeugnis präsentiert wurde. Dieser Befund konnte allerdings nur bei Individuen mit gering ausgeprägter Präferenz für die Produktattribute festgestellt werden. Scarpi (2004, S. 315 ff.) gelang zu der Erkenntnis, dass die Bewertung der Qualität des zuerst offerierten Guts besser ausfällt als die des zuletzt dargebotenen. Diese Einsicht konnte durch Studien in der Online-Umgebung mit längeren Ergebnislisten bestätigt werden; verschiedene Autoren fanden auch bei Trefferlisten von Suchmaschinen (Pan et al. 2007, S. 814 ff.) und bei hintereinander aufgelisteten „links“ (Murphy et al. 2006, S. 525 ff.; Shami et al. 2008, S. 1091) eine stärkere Präferenz für die anfänglich aufgelisteten Treffer. Kardes u. Kalyanaram (1992, S. 347 ff.) liefern Belege für Rangordnungseffekte bei der Markteinführung neuer Produkte; danach zeichnen sich Erzeugnisse, die zuerst auf dem Markt eingeführt wurden, durch eine größere Wahlhäufigkeit aus als später präsentierte. Zudem zeigen Diehl u. Zaubermann (2005, S. 892 ff.), dass die Anordnung von Objekten nach einem bestimmten Kriterium aufsteigend oder absteigend einen Einfluss auf die von Individuen erlebte Qualität des gewählten Objekts besitzt.

Trotz dieser Beiträge zur Wirkung der Rangordnung auf das individuelle Entscheidungsverhalten sind drei Fragen zu diesem Thema noch nicht beantwortet:

(1) Zunächst ist zu klären, inwiefern sich die Wahlwahrscheinlichkeit für ein Produkt in einer Rangreihung beeinflussen lässt. In allen bisherigen Studien diente die Produktpräferenz als abhängige Variable (z. B. Day 1969; Dean 1980; Gierl u. Höser 2002; Scarpi 2004). Ob und inwieweit sich Rangordnungseffekte auch auf die Produktwahl ${ }^{4}$ auswirken, soll in dieser Arbeit überprüft werden.

(2) Sofern sich die Wirkung des Rangplatzes auf das Entscheidungsverhalten als robust erweist, ist zu klären, welcher Rangplatz die stärkste Produktpräferenz evoziert. Da bislang vor allem Listen bestehend aus zwei Objekten betrachtet wurden, konnte lediglich die Frage beantwortet werden, ob das Erzeugnis auf dem ersten oder das auf dem zweiten Platz aus Kundensicht attraktiver ist. Ein Blick auf das Marktgeschehen (insbesondere im Internet) zeigt, dass Listen mit mehr als zwei Optionen gängige Praxis sind; man denke etwa an Google, wo per ,default“ zehn Treffer auf einer Seite präsentiert sind. In diesem Zusammenhang sollen zudem Rangordnungseffekte in Listen untersucht werden, die nach einem Kriterium geordnet sind. Listen dieser Art sind bei Internet-Börsen häufig zu finden.

(3) Ferner ist zu klären, ob es einem Unternehmen gelingt, die Präferenz der Kunden für ein Erzeugnis, das sich auf einem bestimmten Rangplatz befindet, zu lenken (etwa durch farbliche Hervorhebung oder durch die Bereitstellung von Informationen über dieses Erzeugnis). Eine Antwort auf diese Frage liefert Aufschluss darüber, inwieweit sich der Einfluss des Rangplatzes, auf dem sich ein Erzeugnis befindet, auf dessen Attraktivität für die Kunden als robust erweist. Hieraus ergeben sich Hinweise über die Möglichkeiten und Grenzen der marketingpolitischen Gestaltung (bzw. Ausnutzung) des Rangplatzeffekts.

Zur Bewältigung dieser Aufgaben liegt eine dreistufige Vorgehensweise nahe: Zunächst sind zentrale theoretische Ansätze zu diskutieren, aus denen sich Hypothesen über den Einfluss der Rangordnung auf das Entscheidungsverhalten ergeben. Im Rahmen einer sich anschließenden empirischen Untersuchung sollen die formulierten Hypothesen überprüft 
werden. Eine Analyse des tatsächlichen Entscheidungsverhaltens erlaubt abschließend die Formulierung von praktischen Hinweisen für die Gestaltung von Produktrangfolgen sowie eine theoretische Diskussion über das Entscheidungsverhalten von Personen bei der Auswahl von Erzeugnissen aus Listen.

\section{Hypothesen über die Wirkung der Produktrangfolge auf das Entscheidungsverhalten}

Will man das Verhalten von Individuen bei der Wahl eines Erzeugnisses aus einer Liste verstehen, spielen mit Blick auf die Literatur zu diesem Thema drei theoretische Zugänge eine Rolle. Aus diesen Ansätzen lassen sich über das interessierende Phänomen Hypothesen ableiten, die sich wechselseitig ergänzen aber auch im Widerspruch zueinander stehen. Eine sich anschließende empirische Untersuchung (Kap. 3) muss Aufschluss über die Relevanz der einzelnen Ansätze liefern.

Zunächst interessiert, ob von der Erinnerung eines Individuums an die einzelnen auf einer Liste präsentierten Güter ein Effekt auf die Wahlhandlung ausgeht. „Primacy“- und „recency“-Effekten zufolge erinnert sich eine Person vor allem an die zuerst und die zuletzt dargebotenen Produkte (Kap. 2.1). Daraufhin kommt die ,prospect“-Theorie in Betracht, die im Widerspruch zu Primacy- und Recency-Effekten steht. Sie basiert im Kern auf der Referenzpunktbezogenheit und der Verlustaversion von Entscheidern. Aus diesem Ansatz lässt sich sowohl die Attraktivität der ersten Option in einer randomisierten Rangreihung als auch die Attraktivität der mittleren Option in einer geordneten Liste ableiten (Kap. 2.2). Abschließend gilt das Augenmerk dem metakognitiven Wissen einer Person bei der Entscheidung für ein Gut aus einer Rangfolge. Dieser „,marketplace metacognition“ zufolge entwickelt eine Person bei der Objektwahl eine metakognitive Vorstellung über die Position, auf der der Anbieter das für sie vermeintlich attraktivste Produkt in der Liste anordnet (Kap. 2.3).

\section{1 „Primacy“- und „recency“-Effekte}

Eine Aufarbeitung der Literatur zeigt, dass die Wirkung der Produktrangfolge auf das Entscheidungsverhalten von Individuen mit lernpsychologischen Befunden erklärt werden kann (Dean 1980, S. 109 ff.). Hierbei spielen insbesondere die dem Konzept des seriellen Positionseffekts subsumierten „primacy“- und „recency“-Effekte eine zentrale Rolle. Beide Phänomene konnten im Zusammenhang mit der Fähigkeit von Individuen, Wortlisten zu erinnern, entdeckt werden. Vielfältige Studien liefern die Erkenntnis, dass die Intensität der Erinnerung an Worte aus einer Liste von deren Position in dieser Liste abhängt (Glanzer u. Cunitz 1966, S. 354 ff.; Terry 2005, S. 155 ff.; Raanaas u. Magnussen 2006, S. 405 ff.). Stets wurden Worte vom Anfang und vom Ende der Liste von den Probanden besonders gut erinnert; der Zusammenhang zwischen der Position eines Wortes in einer Rangordnung und der Fähigkeit einer Person, sich an diesen Begriff zu erinnern, verläuft somit u-förmig (Deese u. Kaufman 1957, S. 182; Murdock 1962, S. 483). Anknüpfend an diese Grundlagenstudien wurden ,primacy“- und „recency“-Effekte auch mehrfach zur Erklärung des Wahlverhaltens von Individuen herangezogen. Auch hier zeigt sich die besondere Attraktivität der ersten und letzten Optionen (Murphy et al. 2006). Aufgrund der 
Analogie dieses Sachverhalts zur Wahl von Erzeugnissen aus einer Rangreihung lässt sich die folgende Hypothese formulieren:

H1: Aus einer Liste mit Produkten wählen Personen die ersten und die letzten Optionen besonders oft aus (u-förmiger Zusammenhang).

\section{2 „Prospect“-Theorie}

Ein weiterer Ansatz zur Erklärung der Wirkung der Produktrangfolge auf das Entscheidungsverhalten der Individuen ist die von Kahneman u. Tversky (1979) entwickelte „prospect“-Theorie (Novemsky u. Kahneman 2005a, 2005b; Thaler 1985, Thaler u. Johnson 1990). Ihr zufolge beurteilt eine Person vorliegende Optionen in Bezug auf einen Referenzpunkt (Schweitzer 1994, S. 460 ff.; Samuelson u. Zeckhauser 1988, S. 14 ff.). Dieser Anker dient dem Entscheider dazu, ein relatives Urteil über alle zur Auswahl stehenden Produkte zu treffen. Als Referenzpunkt kommt beispielsweise ein Erzeugnis der präsentierten Gütermenge, ein mittleres Produkt im Markt oder ein fiktives, das aus den Erfahrungen und dem Wissen des Individuums gebildet wurde, in Betracht.

Darüber hinaus sind Individuen verlustavers (Tversky u. Kahneman 1991, S. 1041 ff.; Abdellaoui et al. 2005, S. 1391 ff.); d. h. negative Abweichungen vom Ankerpunkt wiegen stärker als positive. Hierzu seien zwei Pkw betrachtet, die sich durch zwei von den Probanden als gleichgewichtig eingestuften Merkmale beschreiben lassen. Das Referenzfahrzeug weist eine PS-Zahl von 100 und einen Benzinverbrauch von $10 \mathrm{~L}$ pro $100 \mathrm{~km}$ auf, wohingegen das zu beurteilende Automobil 80 PS besitzt und $8 \mathrm{~L}$ auf $100 \mathrm{~km}$ verbraucht. Objektiv gesehen entspricht die negative Abweichung des betrachteten Pkw bei der PS-Zahl exakt der positiven Abweichung beim Benzinverbrauch. Da ein Individuum negative Erlebnisse stärker empfindet als im Ausmaß identische positive, müsste der zu beurteilende Pkw beim Benzinverbrauch besonders gut abschneiden (z. B. nur 5 oder $6 \mathrm{~L}$ pro km verbrauchen), um für eine Wahl in Betracht zu kommen.

\section{- Erster Platz als Referenzpunkt}

Zahlreiche empirische Untersuchungen aus verschiedenen wissenschaftlichen Traditionen liefern Hinweise, dass das erste Erzeugnis aus einer Liste für viele Kunden als Referenzpunkt fungiert (Kardes u. Sanbonmatsu 1993, S. 42; Gierl u. Höser 2002, S. 5 ff.; Polak et al. 2008). Bei Betrachtung einer Liste bestimmen Individuen zunächst ein Objekt als Anker, um danach die anderen Objekte damit zu vergleichen. Sofern dieser Anker nicht durch bestimmte Rückschlüsse festgelegt werden kann (z. B. das leistungsfähigste oder das preisgünstigste Objekt), kommt die Zugänglichkeitsheuristik zum Einsatz (Mussweiler 2003, S. 474). Hierbei greifen die Personen das am schnellsten und leichtesten zugängliche Objekt auf und erheben es zum Anker. Gerade bei einer randomisierten Liste (d. h. die Objekte sind nicht im Hinblick auf ein Kriterium sortiert) ist es nicht ohne Weiteres möglich, einen Anker zu finden. Folglich ist zu erwarten, dass viele Individuen das erste Objekt als Referenz heranziehen. Aufgrund der individuellen Verlustaversion müssen alle folgenden Erzeugnisse, die auf einem Merkmal eine schlechtere Ausprägung aufweisen, auf einem anderen einen deutlich besseren Wert besitzen, um für die Wahl in Betracht zu kommen. Daher gilt die folgende Hypothese, die als Alternativhypothese zu H1 zu sehen ist: 
H2: Bei einer randomisierten Liste wählen die Individuen das Objekt auf dem ersten Listenplatz besonders oft.

- Mittlerer Platz als Referenzpunkt

Simonson (1989) stellt in einer empirischen Untersuchung fest, dass Individuen in bestimmten Entscheidungssituationen die mittlere Option in einer Liste von Erzeugnissen bzw. Merkmalsausprägungen als attraktiv erachten. Zur Erklärung dieses „,compromise“Effekts existieren in der Literatur verschiedene Ansätze, die im Kern auf der ,prospect“Theorie und der damit verbundenen Vorstellung der Verlustaversion basieren (Simonson 1989, S. 171 ff.; Simonson u. Tversky 1992, S. 289 ff.). Dabei dient die mittlere Position als Referenz (Simonson 1989, S. 171), was empirisch bereits mehrmals bestätigt wurde (Kivetz et al. 2004; Sheng et al. 2005; Chernev 2004; Prelec et al. 1997). Im Gegensatz zur randomisierten Liste erhält das Individuum bei einer geordneten Liste die Information, dass an den beiden Enden die im Hinblick auf ihre Beschaffenheit extremen Produkte (z. B. Gut A mit niedrigem Preis bei niedriger Qualität bzw. Erzeugnis B mit hohem Preis bei hoher Qualität) angesiedelt sind. Diese Sortierung ermöglicht eine Bestimmung des Ankers aufgrund von Rückschlüssen (Mussweiler 2003, S. 474). Es konnte gezeigt werden, dass Individuen den Rückschluss ziehen, beim mittleren Objekt handle es sich um das von allen Kunden am häufigsten gekaufte (Prelec et al. 1997, S. 123 ff.; Wernerfelt 1995, S. 627 ff.). Deshalb bietet es sich an, dieses als Vergleichsstandard zu wählen. Da ein Individuum negative Abweichungen vom Ankerpunkt stärker empfindet als im Ausmaß identische positive, müsste Gut A (im Vergleich zum Referenzprodukt) einen besonders niedrigen Preis und Erzeugnis B (relativ zum Ankerprodukt) eine besonders hohe Qualität aufweisen, um für eine Wahl in Betracht zu kommen (Prelec et al. 1997, S. 118 ff.). Folglich lässt sich die folgende Hypothese, die eine Alternativhypothese zu H1 darstellt, formulieren:

H3: Bei einer geordneten Liste wählen die Individuen die Option auf dem mittleren Listenplatz besonders oft.

\subsection{Konzept der „,marketplace metacognition“}

Individuen stellen regelmäßig Überlegungen an, ob und inwieweit die zur Wahl stehenden Erzeugnisse ihnen nützlich sind oder lediglich den Interessen der Unternehmen dienen (Friestad u. Wright 1994, S. 4 ff., 1995, 1999, S. 186). Die Einschätzung der Nachfrager bezüglich der Absichten und des Verhaltens der Anbieter kommt in der ,marketplace metacognition“" zum Ausdruck (Wright 2002, S. 679 ff.; Campbell u. Kirmani 2000, S. 70 ff.; Brown u. Krishna 2004, S. $530 \mathrm{ff}$.). Dieses Urteil reflektiert „... everyday individuals' thinking about market-related thinking. This includes people's beliefs ... about other people's beliefs on those topics as these beliefs pertain to the specific domain of marketplace cooperation and manipulation ..." (Wright 2002, S. 677). Im Kern lässt sich die „,marketplace metacognition“" als Einschätzung des Kunden über die Redlichkeit eines Unternehmens charakterisieren.

Einen Pol des Spektrums möglicher Kundeneinschätzungen bildet das Urteil, die vorliegende Rangreihe sei allein mit dem Ziel gestaltet worden, den Umsatz bzw. Gewinn des 
Unternehmens zu optimieren. In diesem Fall erzeugt die Offerte Misstrauen oder Reaktanz, da die Nachfrager davon ausgehen, der Anbieter wolle ihr Entscheidungsverhalten in seinem Sinne beeinflussen. Der andere Pol bringt die Vorstellung der Individuen zum Ausdruck, die Präsentation der Erzeugnisse trage dazu bei, den Entscheidungsprozess zu erleichtern und zu beschleunigen. Zudem könne der Einzelne durch Unterstützung des Unternehmens im Entscheidungsprozess eher zu einem Produkt gelangen, das seine Bedürfnisse umfassend befriedigt. Hier dominiert die Überzeugung, der Anbieter hätte die Verwendungszwecke der Nachfrager vor Augen und würde die Produktliste nach diesem Kriterium gestalten. Wo sich das Unternehmen aus Sicht der Kunden auf diesem Kontinuum befindet, hängt von dessen Vertrauenswürdigkeit ab (Jain u. Posavac 2001, S. 173 ff.).

Ein Blick auf das Marktgeschehen zeigt vor allem zwei Spielarten der Präsentation von Produktlisten, bei denen die Vermutung nahe liegt, dass Individuen metakognitive Überlegungen anstellen: (1) In zahlreichen Listen sind einzelne Objekte hervorgehoben, etwa durch eine farbliche Kennzeichnung. Beispiele hierfür bilden Auktionsportale, Ergebnislisten von Suchmaschinen oder Verkaufsbörsen (fireball.de, ricardo.ch). Hier könnten die Betrachter dazu stimuliert werden, über den Grund der Hervorhebung nachzudenken. (2) Einige Unternehmen präsentieren die Urteile ihrer Kunden über die zur Wahl stehenden Produkte (amazon.de, otto.de, weltbild.de, cede.ch). Auch in diesem Fall ist zu erwarten, dass Nachfrager diese Informationen nicht nur zur Kenntnis nehmen, sondern über die Absicht des Anbieters nachdenken. Daher liegt folgende Hypothese auf der Hand:

H4a: Sind einzelne Produkte in einer Liste hervorgehoben oder sind persuasive Informationen über einzelne Erzeugnisse präsentiert, geben mehr Personen an, eine Vorstellung über die Anordnung des besten Objekts in der Produktliste zu besitzen als in der entsprechenden Liste ohne diese Einflussfaktoren.

Zudem liegen Hinweise vor, dass die Hervorhebung einzelner Objekte und die Vermittlung persuasiver Informationen die Entscheidungssituation insofern verändern, als in den Augen der Nachfrager die Absicht des Anbieters zu deren Beeinflussung zum Vorschein kommt (Darke u. Ritchie 2007, S. 115 ff.; Brown u. Krishna 2004, S. 531 ff.). Vorsicht und Misstrauen prägen das Entscheidungsverhalten der Individuen mit der Folge, dass die zuvor postulierte Heuristik, die Wahrnehmung und Beurteilung der in der randomisieren Liste dargebotenen Objekte an dem auf dem ersten Rangplatz präsentierten Gut zu orientieren (vgl. Hypothese 2), nicht mehr wirksam ist. Folglich soll die folgende Hypothese getestet werden:

$H 4 b$ : Der in Hypothese 2 postulierte Effekt, dass bei einer randomisierten Liste die Individuen das Produkt auf dem ersten Listenplatz besonders oft wählen, verschwindet, sofern das Unternehmen einzelne Objekte hervorhebt oder persuasive Informationen vermittelt.

Um sicher zu stellen, dass sowohl bei der Hervorhebung von Objekten als auch bei der Bereitstellung persuasiver Informationen metakognitive Vorstellungen im Spiel sind, soll eine zweite abhängige Variable betrachtet werden. Erkenntnissen von Payne et al. (1993, S. 73) sowie Kahneman und Frederick (2002, S. 51 ff.) zufolge führt die Aktivierung metakognitiver Überlegungen zu einer Reduktion der Entscheidungsqualität. Ein Individuum richtet seine Wahlhandlung nicht an seinen Bedürfnissen aus, sondern entscheidet auf 
Basis von Vertrauens- oder Misstrauensheuristiken. Damit schließt es bestimmte Produkte von vornherein (aufgrund von Misstrauen) aus. Bei der Entscheidungsqualität geht es nicht um gute oder schlechte (bzw. richtige oder falsche) Entscheidungen im objektiven Sinn, sondern allein um das Ausmaß, in dem die getroffenen Entscheidungen den eigenen Präferenzen entsprechen. So verstanden sollte bei Personen, deren Entscheidungen auf metakognitiven Vorstellungen basieren, die Entscheidungsqualität geringer sein, als bei Individuen, die sich nicht von solchen Heuristiken leiten lassen und ihre Wahl unter Berücksichtigung ihrer Präferenzen treffen. Deshalb soll die folgende Hypothese überprüft werden:

H4c: Eine farbige Hervorhebung einzelner Produkte oder die Bereitstellung persuasiver Informationen führt zu einer Reduktion der Entscheidungsqualität.

Anknüpfend an diese Hypothese ist zu klären, inwiefern eine Aktivierung metakognitiver Vorstellungen tatsächlich die Entscheidungsqualität reduziert. Hierbei gilt das $\mathrm{Au}-$ genmerk der mediierenden Wirkung der von den Individuen postulierten metakognitiven Überlegungen auf den Zusammenhang zwischen der Hervorhebung von Objekten bzw. der Persuasion durch Informationen und der Entscheidungsqualität (vgl. zur Thematik von Mediationseffekten: Baron u. Kenny 1986). Sofern die Aktivierung metakognitiver Vorstellungen die Ursache für die Verminderung der Entscheidungsqualität ist, müsste der Effekt der Hervorhebung bzw. Persuasion auf die Entscheidungsqualität durch die metakognitiven Überlegungen über die Listenanordnung mediiert werden. Daher ist die folgende Hypothese zu testen:

H4d: Die metakognitiven Vorstellungen mediieren den Zusammenhang zwischen der Hervorhebung von Objekten bzw. der Persuasion durch Informationen und der Entscheidungsqualität.

Abschließend gilt das Interesse der Wirkung metakognitiver Vorstellungen auf das Entscheidungsverhalten der Individuen. Erste Anhaltspunkte hierzu legen die Vermutung nahe, dass die metakognitiven Überlegungen über den Grund der Hervorhebung von Objekten durch den Anbieter einen Einfluss auf die Entscheidungen der Nachfrager ausüben (Brown u. Krishna 2004, S. 531 ff.). Hegt eine Person, der eine Liste mit farbig hervorgehobenen Produkten vorliegt, die Überzeugung, die markierten Objekte seien die besten für sie (Vermutung der positiven Beeinflussung), entscheidet sie sich für das hervorgehobene Gut. Dominiert hingegen die Idee, dass ein Produkt markiert ist, weil es der Hersteller oder Händler absetzen möchte (Vermutung der negativen Beeinflussung), greift das Individuum zu einem nicht hervorgehobenen Erzeugnis. Insofern gilt folgende Hypothese:

H4e: Nachfrager, die eine negative Beeinflussung durch den Anbieter vermuten, wählen eher nicht hervorgehobene Optionen; Personen, die eine positive Beeinflussung durch das Unternehmen vermuten, wählen eher hervorgehobene Optionen.

Die postulierten Hypothesen sollen im Folgenden im Rahmen einer empirischen Untersuchung überprüft werden. 


\section{Empirische Untersuchung}

\subsection{Untersuchungsdesign und Datenerfassung}

Zur Überprüfung der zuvor formulierten Hypothesen bot sich ein Experiment im Automobilmarkt an. Hierzu wurde auf die Gebrauchtwagenbörse eines Automobilunternehmens zurückgegriffen. Alle Pkw-Hersteller besitzen Börsen dieser Art, auf denen gebrauchte Firmenfahrzeuge gehandelt werden. Interessiert sich ein Individuum für ein solches Automobil, kann es unter Rückgriff auf (nach Hersteller variierenden) Suchkriterien Fahrzeuge finden, die seinen Wünschen entsprechen. $\mathrm{Zu}$ diesen Kriterien gehören beispielsweise die Baureihe (z. B. A4 von Audi), ein Spektrum der gewünschten PS-Zahl, die maximal gefahrenen Kilometer und ein Preiskorridor.

Um die Komplexität der Informationen über die Automobile bewältigbar zu halten, galt im Experiment der Fokus einer Baureihe (Audi A4). Pkw diesen Typs wurden mit einem Bild sowie Angaben über den Preis, die PS-Zahl und den Kilometerstand präsentiert. Jedem Probanden lag eine Liste vor, die aus sieben Automobilen bestand. Der Umfang der Liste wurde experimentell nicht verändert, weil der Kooperationspartner darauf bestand, die empirische Untersuchung im Hinblick auf die zu generierenden Konditionen überschaubar zu halten. Zur Überprüfung der Hypothesen sind die in Tab. 1 dargestellten sechs Konditionen erforderlich. Hierbei handelt es sich um ein reduziertes Design, da auf Wunsch des Kooperationspartners die Fallzahl nicht beliebig ausgeweitet werden

Tab. 1: Zuordnung der Konditionen zu den Hypothesen

\begin{tabular}{|c|c|c|c|c|}
\hline \multicolumn{4}{|c|}{ Kondition } & \multirow[t]{2}{*}{ Hypothesen } \\
\hline Nr. & $\begin{array}{l}\text { Reihenfolge der } \\
\text { präsentierten } \\
\text { Pkw }\end{array}$ & $\begin{array}{l}\text { Anordnung der } \\
\text { präsentierten } \\
\text { Pkw }\end{array}$ & $\begin{array}{l}\text { Persuasive } \\
\text { Informationen } \\
\text { bzw. farbliche } \\
\text { Hervorhebung }\end{array}$ & \\
\hline (1) & zufällig & untereinander & - & $\begin{array}{l}\mathrm{H} 1, \mathrm{H} 2, \\
\mathrm{H} 4 \mathrm{a}, \mathrm{H} 4 \mathrm{~b}, \\
\mathrm{H} 4 \mathrm{c}\end{array}$ \\
\hline (2) & $\begin{array}{l}\text { geordnet nach Merkmal } \\
\text { (Preis, PS-Zahl, } \\
\text { Kilometerstand) }\end{array}$ & untereinander & - & H1, H3 \\
\hline (3) & zufällig & nebeneinander & - & $\mathrm{H} 1, \mathrm{H} 2$ \\
\hline (4) & $\begin{array}{l}\text { geordnet nach Merkmal } \\
\text { (Preis, PS-Zahl, } \\
\text { Kilometerstand), }\end{array}$ & nebeneinander & - & H1, H3 \\
\hline (5) & zufällig & untereinander & $\begin{array}{l}\text { Automobile } 1 \text { bis } 3 \\
\text { farblich } \\
\text { hervorgehoben }\end{array}$ & $\begin{array}{l}\mathrm{H} 4 \mathrm{a}, \mathrm{H} 4 \mathrm{~b} \\
\mathrm{H} 4 \mathrm{c}, \mathrm{H} 4 \mathrm{~d} \\
\mathrm{H} 4 \mathrm{e}\end{array}$ \\
\hline (6) & zufällig & untereinander & $\begin{array}{l}\text { Präsentation von } \\
\text { Nutzerempfehlungen } \\
\text { für die Fahrzeuggattung } \\
\text { A4 (nicht für einzelne } \\
\text { A4-Modelle) }\end{array}$ & $\begin{array}{l}\mathrm{H} 4 \mathrm{a}, \mathrm{H} 4 \mathrm{~b} \\
\mathrm{H} 4 \mathrm{c}, \mathrm{H} 4 \mathrm{~d} \\
\mathrm{H} 4 \mathrm{e}\end{array}$ \\
\hline
\end{tabular}


Instruktion: Bitte stellen Sie sich vor, Sie möchten heute einen gebrauchten Audi A4 kaufen und haben die folgenden Fahrzeuge zur Auswahl. Für welches Auto entscheiden Sie sich?

Die Autos sind geordnet nach der PS-Zahl
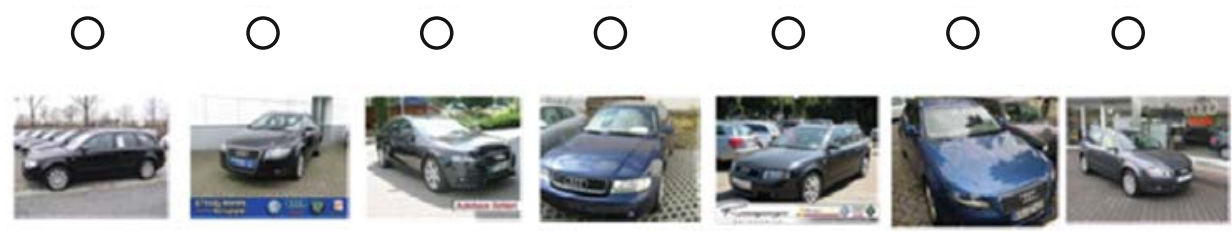

17.900 Euro

179 PS

$159000 \mathrm{~km}$

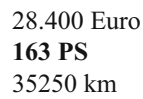

Nr: 1

2

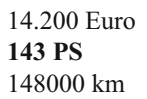

3

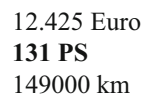

5

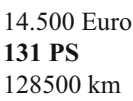

6

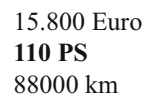

7
23.500 Euro 110 PS $13000 \mathrm{~km}$

Erläuterung zur Abbildung:

Um Konfundierungseffekte, die durch die Fotos (z. B. durch die Beleuchtung, die Farbe der Autos, oder das Logo eines Autohauses) oder durch aggregierte Präferenzen für bestimmte Merkmalsausprägungen (z. B. eine starke Präferenz bei allen Befragten für eine hohe PS-Zahl) entstehen, eliminieren zu können, wurde in einer separaten Untersuchungsbedingung die Wahlhäufigkeit der hier präsentierten Auto-Modelle erfasst und in aggregierter Form als Kontrollvariable verwendet (vgl. Abschn. 3.2.1).

Abb. 1: Beispiel für eine präsentierte Auswahlliste (Kondition 4)

konnte. Dabei wurde für jede Listensortierung eine Kondition mit Darstellung der Objekte untereinander (Konditionen 1 und 2) und eine mit entsprechender Darbietung nebeneinander (Konditionen 3 und 4) erstellt. Diese Unterscheidung ist aus praktischer Perspektive bedeutsam, weil im Internet eben solche Listen zu finden sind. Die Variation der Listenanordnung (untereinander vs. nebeneinander) wurde gewählt, um die Vielfalt des Marktes nachzubilden. Aus den dargestellten Theorien lassen sich keine separaten Hypothesen über diese verschiedenen Darstellungsformen ableiten. Deshalb wurden die untereinander und nebeneinander angeordneten Listen bei der Überprüfung der jeweiligen Hypothesen (randomisiert vs. geordnet) zusammengefasst.

Abbildung 1 zeigt die in jeder Kondition verwendeten Fahrzeuge, die in dieser Grafik im Hinblick auf ihre PS-Zahl nebeneinander angeordnet sind (vgl. Kondition 4). Die sechs Konditionen wurden mit der Gebrauchtwagenbörse eines Pkw-Unternehmens verknüpft. Personen, die sich auf der Homepage dieser Börse befanden, lud man ein, am Experiment teilzunehmen. In allen Konditionen bestand die Aufgabe der Probanden darin, analog zu einer realen Entscheidungssituation das bevorzugte Fahrzeug anzuklicken. Im Anschluss mussten Personen in jenen Konditionen, die zur Überprüfung der „marketplace metacognition" gebildet wurden, noch Fragen beantworten. Diese verkörpern aus der Literatur übernommene Messvariablen zur Erfassung der zentralen Konstrukte „,metakognitive Vorstellungen“ und „Entscheidungsqualität“. Abschließend wurden einige demographische Variablen erhoben.

Bei der Analyse wurde der Datensatz zunächst auf Ausreißer untersucht. Dabei schloss man die Nennungen jener Personen aus, die weniger als 5 min für die Studie benötigten oder 
die keine der Fragen beantworteten. Im Einklang mit Kenntnissen aus anderen empirischen Untersuchungen ist zu vermuten, dass es diesen Individuen lediglich darum ging, an der Verlosung der ausgelobten Preise teilzunehmen. Eine Sensitivitätsanalyse zeigt, dass sich die Resultate bei einer Änderung dieser Schwelle auf 4 oder 6 min nicht verändern. Nach dieser Datenbereinigung verblieben von den ursprünglich 1.730 Probanden noch 1.114 in der Stichprobe (ca. 200 pro Kondition). Die Befragungspersonen benötigten durchschnittlich 9,65 min, um das gewünschte Fahrzeug auszuwählen und den Fragebogen zu beantworten. Für die eigentliche Pkw-Wahl nahmen sie sich im Mittel 1,60 min Zeit. 89,7\% von ihnen waren männlich, 10,3\% weiblich. Als Anreiz für die Teilnahme am Experiment wurden unter allen Befragten sechs Lifestyle-Artikel des Kooperationspartners verlost.

\subsection{Vorstudien und Variablendefinition}

Zur Beantwortung der eingangs aufgeworfenen Fragen im Rahmen einer empirischen Untersuchung sind drei Vorarbeiten durchzuführen. Zunächst ist es erforderlich, die betrachteten Pkw gleichwertig im Hinblick auf die sie beschreibenden Merkmale zu gestalten (Abschn. 3.2.1). Damit soll für die weitere Analyse eine Konfundierung zwischen ihrer Beschaffenheit und dem Rangplatz bei der Erklärung des Wahlverhaltens vermieden werden. Daraufhin gilt das Augenmerk einer Operationalisierung der für die Studie zentralen Konstrukte (vgl. Abschn. 3.2.2). Schließlich ist aus mathematisch-statistischer Perspektive ergänzend zu den sieben existierenden Kategorien eine Referenzkategorie zu definieren (Abschn. 3.2.3). Sie dient dazu, die Variation der Anzahl gewählter Pkw entlang der sieben Kategorien im Hinblick auf die Signifikanz zu beurteilen.

\subsubsection{Konstruktion der Fahrzeuge}

Um die Wirkung des Rangplatzes eines Pkw auf das Wahlverhalten der Individuen zu erforschen, ist es unerlässlich, die betrachteten Autos in ihrer Beschaffenheit möglichst gleichwertig zu gestalten. Zu diesem Zweck sind die Effekte der die Fahrzeuge beschreibenden Variablen (Preis, PS-Zahl, Kilometerstand) auf die Präferenzurteile zu ermitteln und für die Analyse zu eliminieren. Hierzu kommt ein Regressionsmodell in Betracht, bei dem der Preis der Pkw als abhängige Variable fungiert; die PS-Zahl und der Kilometerstand verkörpern die unabhängigen Größen. Auf Basis der entsprechenden Daten über 39 Fahrzeuge, die im Rahmen einer Vorstudie in realen Gebrauchtwagenbörsen erhoben wurden, lautet die Regressionsgleichung wie folgt:

$$
\text { Preis }=9878,00+136,00 \cdot \text { PS-Zahl }-0,1 \cdot \text { Kilometerstand }\left(\mathrm{R}^{2}=0,72\right)
$$

Dieser Gleichung zufolge geht eine Steigerung der PS-Zahl um eine Einheit mit einer Preiserhöhung um $136 €$ einher $(\mathrm{t}=4,72 ; \mathrm{p}<0,01)$. Erhöht sich die Anzahl der gefahrenen Kilometer um eine Einheit, führt dies zu einem um 0,10 € niedrigeren Fahrzeugpreis $(\mathrm{t}=-6,30 ; \mathrm{p}<0,01)$. Mit diesen Variablenrelationen ließen sich bei jedem Automobil der Hauptstudie die PS-Zahl und der Kilometerstand variieren und der entsprechende Preis mit Blick auf die Regressionsgleichung ermitteln. Folglich ist davon auszugehen, dass das Preis-Leistungs-Verhältnis bei allen den Probanden präsentierten Fahrzeugen 
(vgl. Abb. 1) nahezu identisch ist und eine Beeinflussung der Fahrzeugwahl durch die Beschaffenheit der Pkw minimiert werden kann.

Trotz der konstruierten Gleichwertigkeit der Autos sind Störeffekte vorstellbar, die die Entscheidung beeinflussen. Solche Einflüsse können beispielsweise aus der Qualität des Pkw-Fotos oder aus einer besonderen Vorliebe der Personen für eine bestimmte Ausprägung eines Merkmals resultieren. Es ist denkbar, dass für zahlreiche Individuen eine hohe PS-Zahl wichtiger ist als ein niedriger Preis. Deshalb wurde eine Kontrollvariable gebildet, die die mittleren Präferenzen aller Probanden für die dargebotenen Automobile erfasst und auf diesem Wege die Störvarianz, die auf diesen „Beliebtheitseffekt" zurückzuführen ist, vereint.

Zur Bestimmung dieser Kontrollvariable definierte man eine zusätzliche Untersuchungsbedingung. Die Probanden, die dieser Bedingung zugewiesen wurden, nahmen nicht an der Hauptuntersuchung teil. Ihre Angaben wurden nur benötigt, um sie in aggregierter Form als Kontrollvariable für die Hauptuntersuchung zu verwenden. Hierzu wurden die sieben betrachteten $\mathrm{Pkw}$ - mit den gleichen Fotos und Attributsausprägungen, die in der Hauptuntersuchung verwendet wurden (vgl. Abb. 1) - gemeinsam mit anderen Fahrzeugen in einem 20 Automobile umfassenden ,choice set“ präsentiert, in dem man alle Objekte per Zufall rotierte. Die Aufgabe der Probanden bestand analog zur Hauptstudie darin, eine hypothetische Kaufentscheidung zu treffen. Aus den summierten Entscheidungen dieser Untersuchungskondition konnten über alle Probanden Beliebtheitswerte für die sieben interessierenden Automobile gebildet werden, wobei die Zufallsrotation die Rangordnungseffekte auf die Pkw-Wahl ausschloss. Da diese Kontrollgröße die generelle Beliebtheit der Pkw, inklusive dem jeweiligen Foto und der jeweiligen Attributsausprägung angibt, kann postuliert werden, dass die in der folgenden Analyse erzielten Ergebnisse in keiner Weise durch die Details der Fotos oder durch die Präferenzen der befragten Personen verzerrt sind, weil in jede Analyse die beschriebene Kontrollvariable aufgenommen wurde.

\subsubsection{Operationalisierung der Konstrukte}

Die zentrale abhängige Variable in dieser Studie bildet die Entscheidung der Personen für ein Auto. Hierzu wurde für jedes Individuum auf jeder der sieben Positionsvariablen erfasst, ob ein Auto gewählt wurde oder nicht.

Des Weiteren erfordert eine Überprüfung der Hypothesen die Erfassung der Entscheidungsqualität. Sie lässt sich als „,consistencies in preference“ oder ,proportion accurate choices“ (Johnson u. Payne 1985, S. 396 ff.) abbilden; für beide Größen liegt ein Repertoire bewährter Messvariablen vor. Wie erinnerlich geht es jedoch nicht um richtige oder falsche Entscheidungen in einem objektiven Sinn (,proportion accurate choices“), sondern um die „consistencies in preference“. Erfasst werden soll das Ausmaß, in dem die getroffenen Entscheidungen den eigenen Präferenzen besser oder schlechter entsprechen. Hiernach gilt eine Entscheidung eines Individuums bezüglich einer Merkmalsausprägung als „,korrekt“, sofern sie mit seinen Präferenzen für diese Ausprägung des Attributs übereinstimmt.

Dazu wurden die Präferenzen jeder Person bezüglich jeder Ausprägung der einzelnen Produktattribute (niedriger Preis, hohe PS-Zahl, niedriger Kilometerstand) mit je einer Likert-Skala erhoben. Zusätzlich zu diesen drei Skalen, die die gewünschten Attributsausprägungen erfassen, war es im Rahmen der Datenanalyse erforderlich, drei Skalen zu bilden, die die tatsächlich gewählten Attributsausprägungen abbilden. Dabei diente 
die Bandbreite der präsentierten Produkte als Basis der Skalenkonstruktion. Sofern eine Person das Auto mit dem Preis $14.200 €$ wählte, führte dies zu einer Kodierung dieser Entscheidung mit dem Wert „2“ auf der Preisskala, weil es sich bei diesem Modell um das zweitgünstigste der angebotenen Autos handelte. So konnten den drei gewünschten Attributsausprägungen die tatsächlich gewählten gegenüber gestellt und diese dann voneinander subtrahiert werden. Hieraus resultiert die Variable „Entscheidungsqualität“, die die Summe der Abweichungen der tatsächlichen Entscheidung von der optimalen (auf den drei Attributen Preis, PS-Zahl, Kilometerstand) zum Ausdruck bringt. Hohe (geringe) Abweichungen zwischen optimaler und tatsächlich getroffener Entscheidung sind ein Indikator für eine niedrige (hohe) Entscheidungsqualität. ${ }^{5}$

Abschließend gilt das Augenmerk der Bereitschaft des Individuums, metakognitives Wissen zu aktivieren (,marketplace metacognition“). Die metakognitiven Vorstellungen wurden in Bedingung 5 durch eine optische Hervorhebung der ersten drei Fahrzeuge in roter Farbe aktiviert. In Bedingung 6 erfolgte die Aktivierung metakognitiver Vorstellungen durch die Präsentation persuasiver Informationen vor der Entscheidungsaufgabe. Hier legte man den Personen positive Kundenmeinungen über die in Abb. 1 dargestellte Fahrzeuggattung (also den A4 insgesamt, nicht separat für einzelne A4-Modelle) vor. So äußerten sich Fahrer des Wagens zum Beispiel in positiver Weise über die Sparsamkeit sowie die gute Beschleunigungsleistung. Beide Konditionen haben das Ziel, einen Eingriff in die Entscheidungssituation zu symbolisieren, von dem erwartet wird, dass er zu einer Verwendung von Heuristiken führt, die bei Misstrauen erlernt wurden.

Die metakognitven Vorstellungen wurden durch die Frage nach der persönlichen Vermutung über die Platzierung der besten Autos in der Liste erfasst. Anhand der Antworten konnten die Probanden in zwei Gruppen unterteilt werden, in solche mit metakognitiven Vorstellungen (sie gaben an, die besten Produkte auf speziellen Listenplätzen zu vermuten) und in jene ohne diese Vorstellung (sie gaben an, keine Vermutung über die Anordnung der besten Produkte zu besitzen oder sie vermuten eine Anordnung der Pkw nach dem Zufallsprinzip).

\subsubsection{Definition einer Referenzkategorie}

Vor der Datenanalyse ist eine Entscheidung über die Referenzkategorie zu treffen, da alle mathematisch-statistischen Maße nur in Bezug zu dieser Referenzposition interpretierbar sind. Würde man eine der untersuchten Positionen (1-7) als Referenzposition bestimmen, wären die Effektstärken einer speziellen Position zwischen verschiedenen Listen nicht vergleichbar, weil sich die Koeffizienten auf jeweils unterschiedliche Referenzgrößen beziehen. Deshalb ist es unerlässlich, eine Referenzposition zu konstruieren, die bei allen untersuchten Listen den gleichen Wert annimmt und daher als unverfälschter Standardwert angesehen werden kann (Position 8). Diese Variable nimmt den Wert jener Wahlhäufigkeit an, die auftritt, sofern keine Positionseinflüsse vorliegen. Der Wert dieser Position 8 entspricht der erwarteten gleichverteilten Wahlhäufigkeit von 1/7. Sie repräsentiert somit das ökonomische Modell; würden alle Personen rational handeln (d. h. die Position würde keinen Effekt ausüben), wäre der Wert der konstruierten Referenzkategorie von 1/7 auf jeder der sieben Positionen zu finden. 


\subsection{Ergebnisse der Untersuchung}

Anknüpfend an die erläuterten Vorarbeiten sollen im Folgenden die Ergebnisse der empirischen Untersuchung verdeutlicht werden. Im Sinne einer Evaluation des Erkenntnisbeitrags dieser Studie liegt es nahe, entsprechend der in Kap. 2 formulierten Hypothesen vorzugehen.

\subsection{1 „Primacy“- und ,,recency“-Effekte}

Zur Überprüfung der Wirkung möglicher ,,primacy“- und ,,recency“-Effekte bei der Wahl eines Pkw aus einer Liste dient Abb. 2. Sie zeigt die Wahlhäufigkeit von Fahrzeugen in Abhängigkeit ihrer Position. Da sich „,primacy“- und „,recency“-Effekte unabhängig von der Sortierung der Liste zeigen sollten, sind in Abb. 2 die Wahlhäufigkeiten aggregiert über alle Konditionen dargestellt.

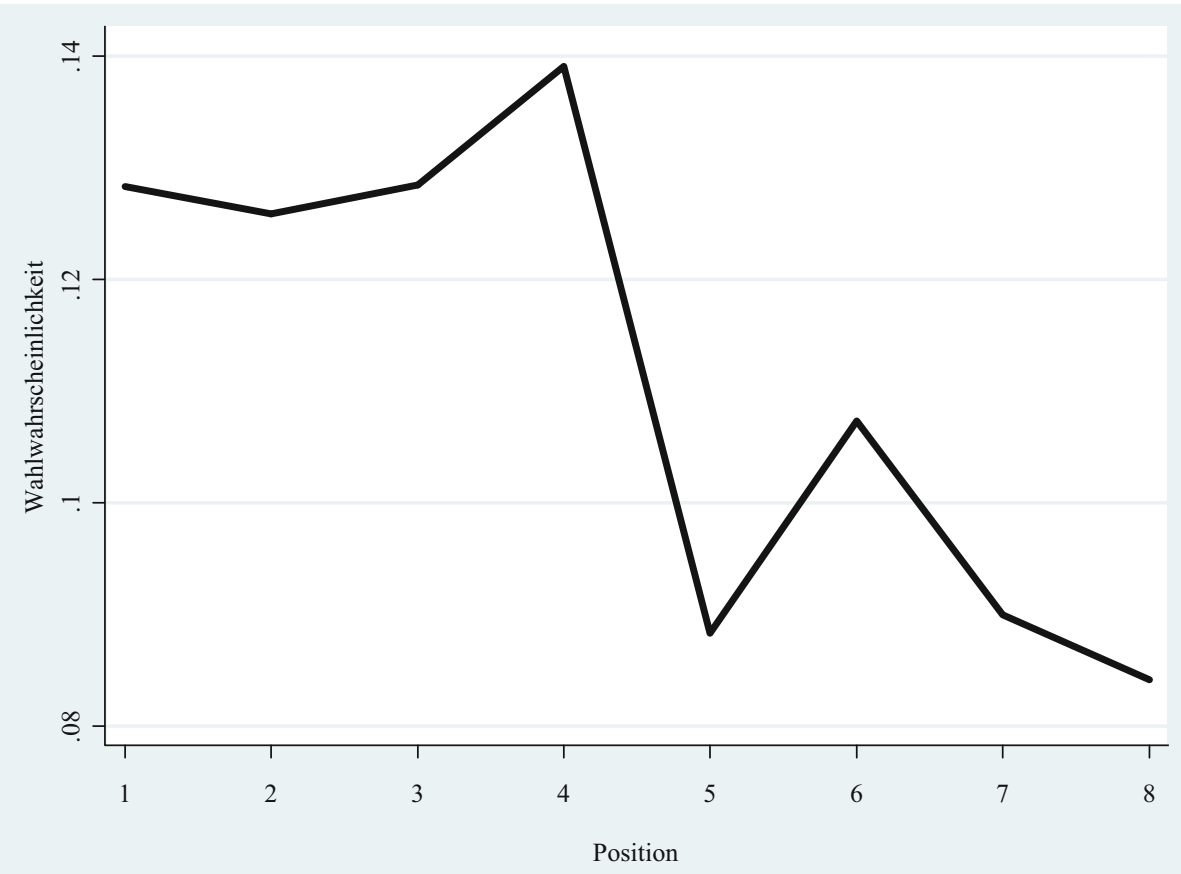

Erläuterung zur Abbildung:

Schwarze Linie $=$ Wahlwahrscheinlichkeit der Bedingungen 1 (die Pkw sind in zufälliger Reihenfolge untereinander dargestellt), 2 (die Präsentation der Fahrzeuge erfolgt untereinander, geordnet nach einem Merkmal), 3 (die Automobile sind in zufälliger Reihenfolge nebeneinander präsentiert) und 4 (man stellt die Pkw nebeneinander, geordnet nach einem Merkmal) gemittelt.

Position $8=\quad$ Konstruierte Referenzposition.

Abb. 2: Wahlwahrscheinlichkeit der jeweiligen Position aggregiert über vier Konditionen 
Bereits ein Blick auf diese Grafik verdeutlicht, dass sich der vermutete u-förmige Zusammenhang nicht bestätigen lässt. Zwar sind die auf dem ersten Listenplatz präsentierten Pkw durch eine hohe Nachfrage gekennzeichnet, diese steigt jedoch bis zum vierten Listenplatz weiter an und sinkt erst danach ab. Würde der ,primacy“- und „recency“-Effekt das Auswahlverhalten determinieren, müsste die Wahlhäufigkeit auf Platz zwei und drei langsam absinken und auf den letzten Listenplätzen wieder langsam ansteigen. Zur Überprüfung der Fragestellung wurde eine logistische Regression mit der abhängigen Variablen ,Entscheidung“" und den unabhängigen Variablen „Position“ und „Beliebtheit“ durchgeführt. Ihre Koeffizienten reflektieren keine Reduktion der Nachfrage auf den ersten Positionen und keinen Anstieg auf den letzten Positionen. Damit ist Hypothese 1, die einen u-förmigen Zusammenhang zwischen der Listenposition und der Auswahlhäufigkeit postuliert, widerlegt.

\subsubsection{Einfluss von Referenzpunkt und Verlustaversion}

\section{- Erster Platz als Referenzpunkt}

Der ,prospect“-Theorie zufolge bildet in randomisierten Listen das auf dem ersten Listenplatz präsentierte Fahrzeug den Referenzpunkt und sollte aufgrund der individuellen Verlustaversion eine hohe Wahlhäufigkeit aufweisen. Abbildung 3 zeigt die Nachfrage für die Pkw in Abhängigkeit ihrer Positionen in den randomisierten Listen. Die Koeffizienten der binären logistischen Regression mit der abhängigen Variablen „Entscheidung “6 ${ }^{\text {und }}$ den unabhängigen Variablen „Position“ und „Beliebtheit“ zeigen, dass das auf der ersten Position dargebotene Automobil die höchste Wahlhäufigkeit aufweist. Ein Vergleich des Koeffizienten $(0,41)$ für diese Position mit den entsprechenden Koeffizienten der Positionen 2 bis 7 (zwischen 0,03 und 0,12) verdeutlicht die Prägnanz dieses Effekts $(z=1,93$; $\mathrm{p}<0,05 ;$ LR Chi $^{2}(\mathrm{df}=8)=718,00 ;$ Prob $>\mathrm{Chi}^{2}=0,00 ;$ Pseudo $\left.\mathrm{R}^{2}=0,14\right)$.

Offenbar führt die Positionierung eines Produkts auf dem ersten Platz bei einer randomisierten Liste zu einer Steigerung seiner Wahlhäufigkeit (im Vergleich zu anderen Positionen), so dass Hypothese 2 vorläufig bestätigt werden kann.

\section{- Mittlerer Platz als Referenzpunkt}

Dem „,compromise effect“ zufolge erachten Individuen nicht nur die erste, sondern auch die mittlere Option in einer Liste von Automobilen als attraktiv. Dieser Effekt sollte vor allem bei geordneten Listen auftreten, da das Kompromissfahrzeug in der Mitte der Liste schnell und leicht zu bestimmen ist und insofern als Referenzpunkt verwendet werden kann. Um die statistische Signifikanz dieses Phänomens zu bestimmen, wurde ein Vergleich der Wahlhäufigkeiten für die Pkw auf den einzelnen Rangplätzen bei geordneten und randomisierten Listen vorgenommen. Die logit-Koeffizienten einer binären logistischen Regression mit der abhängigen Variablen „Entscheidung für die mittlere Position“ und den unabhängigen Variablen „Listenanordnung (zufällig vs geordnet)“ und „Beliebtheit" (Abb. 4) verdeutlichen, dass Probanden die Fahrzeuge auf der mittleren Position (Position 4) in geordneten Listen deutlich häufiger wählen als in randomisierten $(z=2,47$; $\mathrm{p}<0,01$; Koeffizient: 0,73). Somit kann der Einfluss des Kompromisseffekts in geordneten Listen als vorläufig belegt betrachtet werden (vgl. Hypothese $3 ; \mathrm{LR} \mathrm{Chi}^{2}(\mathrm{df}=2)=65,39$; Prob $>\mathrm{Chi}^{2}=0,00 ;$ Pseudo $\mathrm{R}^{2}=0,12$ ). 


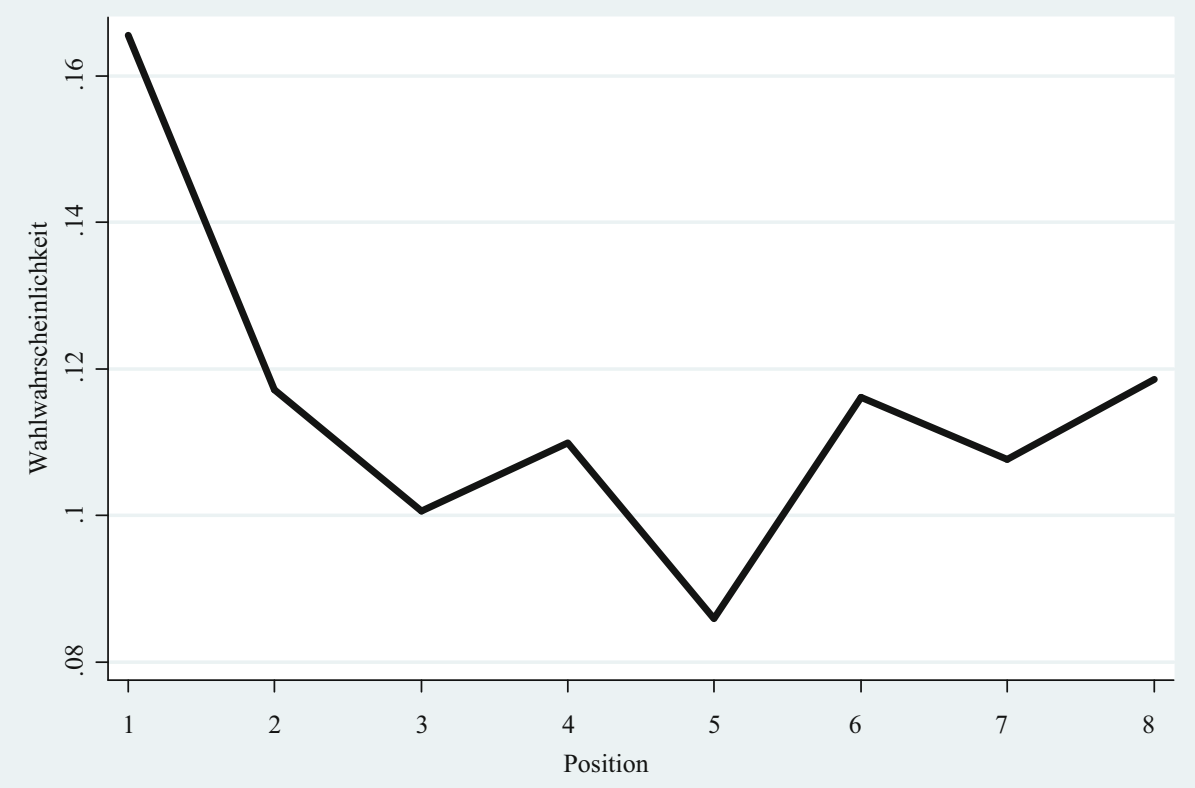

Erläuterung zur Abbildung:

Schwarze Linie = Wahlwahrscheinlichkeit der Bedingungen 1 (die Pkw sind in zufälliger Reihenfolge untereinander dargestellt) und 3 (die Automobile sind in zufälliger Reihenfolge nebeneinander präsentiert) gemittelt.

Position $8=$ Konstruierte Referenzposition.

Abb. 3: Wahlwahrscheinlichkeiten bei randomisierten Listen

Um den ,compromise“-Effekt zu bestätigen, wurde untersucht, ob Individuen mittelpreisige Pkw in geordneten Listen häufiger erwerben als in randomisierten. Dazu teilte man die betrachteten Fahrzeuge in zwei Klassen - in extreme und in durchschnittliche Automobile - auf. Als durchschnittlich galten jene Pkw, die auf den drei Kriterien (Preis, PS-Zahl, Kilometerstand) mittlere Ausprägungen aufwiesen; es handelte sich um die Fahrzeuge mit den Nummern 5, 6 und 7 (siehe Abb. 1). Eine Kreuztabelle zeigt, dass Individuen diese drei Fahrzeuge in geordneten Listen in 9,60\% aller Fälle wählten; der entsprechende Wert für die randomisierten Listen lautet 5,95\%. Ein Chi-Quadrat-Test verdeutlicht, dass die Differenz $(3,65 \%)$ deutlich von Null abweicht $\left(\mathrm{Chi}^{2}(\mathrm{df}=1)=15,48 ; \mathrm{p}<0,01\right)$. Damit liegt eine weitere Bestätigung für Hypothese 3 vor.

\subsubsection{Bedeutung der ,,marketplace metacognition “}

Vor einer Analyse der Wirkung der „marketplace metacognition“ auf das Wahlverhalten interessiert, inwieweit es möglich ist, durch eine farbige Hervorhebung der Pkw oder durch die Präsentation persuasiver Informationen vor der Entscheidung die metakognitiven Vorstellungen über eine spezifische Listenanordnung zu aktivieren. Eine Auswertung der 


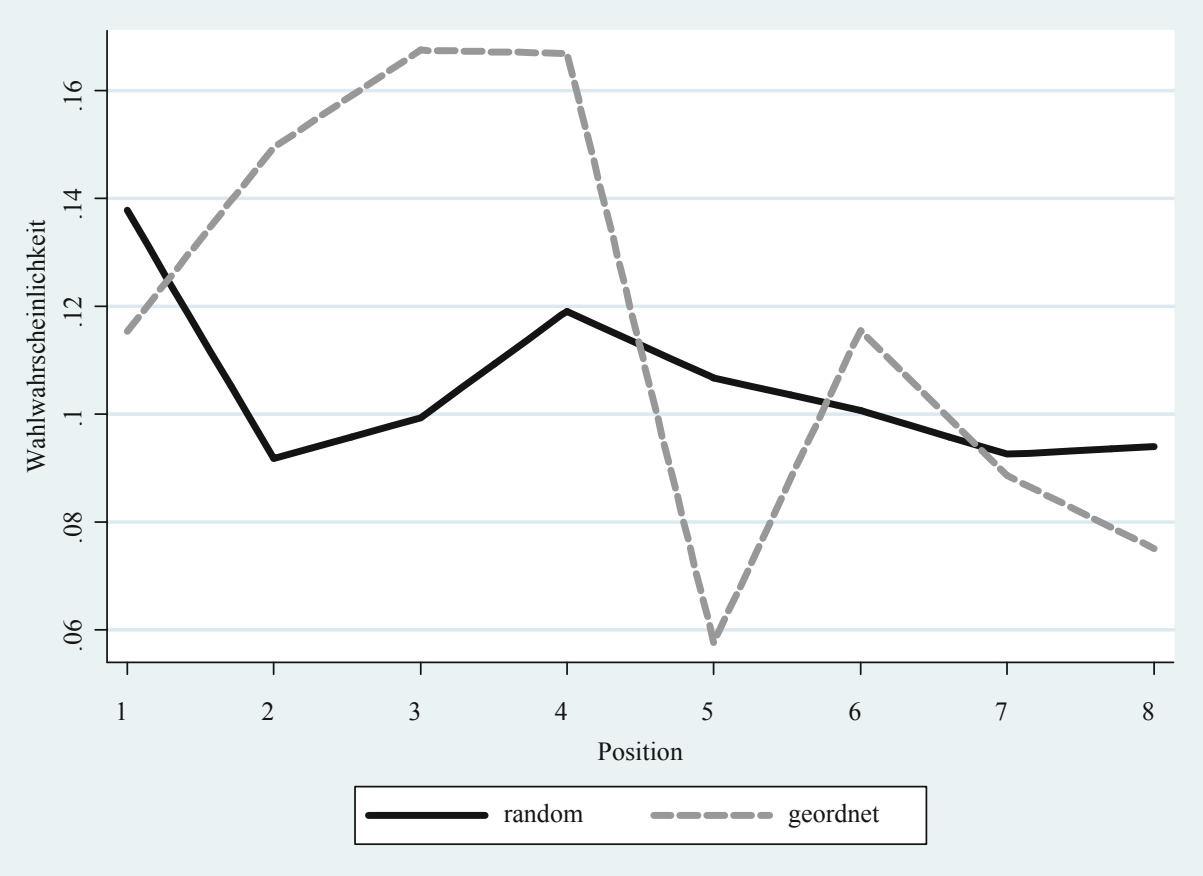

\section{Erläuterung zur Abbildung:}

Schwarze Linie = Wahlwahrscheinlichkeit der Bedingungen 1 (die Pkw sind in zufälliger Reihenfolge untereinander dargestellt) und 3 (die Automobile sind in zufälliger Reihenfolge nebeneinander präsentiert) gemittelt.

Gestrichelte Linie $=$ Wahlwahrscheinlichkeit der Bedingungen 2 (die Präsentation der Fahrzeuge erfolgt untereinander, geordnet nach einem Merkmal) und 4 (man stellt die Pkw nebeneinander, geordnet nach einem Merkmal) gemittelt.

Position 8

$=$ Konstruierte Referenzposition.

Abb. 4: Vergleich der Wahlwahrscheinlichkeiten bei randomisierten und geordneten Listen

erhobenen Daten in einer Kreuztabelle zeigt, dass bei der farbigen Hervorhebung der ersten drei Autos im Vergleich zu einer Kontrollgruppe 8,53\% mehr Personen eine Vermutung über die Anordnung der Automobile in der Liste hegen $\left(\mathrm{Chi}^{2}(\mathrm{df}=1)=37,22 ; \mathrm{p}<0,01\right)$. Auch bei der mit persuasiven Informationen über die Fahrzeuge konfrontierten Gruppe ist dieser Effekt feststellbar; hier beträgt der Unterschied zwischen der Experimental- und Kontrollgruppe zwar nur 2,59\%, was sich jedoch immer noch auf dem 10\%-Niveau als statistisch signifikant erweist $\left(\mathrm{Chi}^{2}(\mathrm{df}=1)=2,72 ; \mathrm{p}<0,09\right)$. Als Kontrollgruppe dienten jeweils jene Individuen, denen die Liste mit den Pkw vorlag, die in zufälliger Anordnung untereinander ohne Eingriff in die Entscheidungssituation präsentiert wurden. Diese Ergebnisse liefern eine vorläufige Bestätigung von Hypothese 4a; die Aktivierung von metakognitiven Vorstellungen durch einen Eingriff in die Entscheidungssituation wurde belegt. 
Darüber hinaus gilt das Augenmerk der Beantwortung der Frage, ob eine Aktivierung metakognitiven Wissens über die Listenanordnung die Attraktivität des auf dem ersten Platz dargebotenen Fahrzeugs reduziert (Hypothese 4b). Die Abb. 5 und 6 zeigen, dass sowohl die Präsentation persuasiver Informationen als auch die farbige Hervorhebung von Pkw den vom ersten Platz ausgehenden Effekt auf das Wahlverhalten eliminiert. Eine binäre logistische Regression zeigt, dass sich bezogen auf die Referenzbedingung der Effekt der persuasiven Information auf die Variable „Entscheidung für Position $1^{\text {" }}$ unter Einschluss der Beliebtheitskontrollvariablen als statistisch signifikant erweist $\left(z=-1,67 ; p<0,09\right.$; Koeffizient: $-0,55 ; \mathrm{LR} \mathrm{Chi}^{2}(\mathrm{df}=2)=40,31 ; \mathrm{Prob}^{2}>\mathrm{Chi}^{2}=0,00$; Pseudo $\mathrm{R}^{2}=0,13$ ). Bezüglich der farbigen Hervorhebung ist der Unterschied in einer entsprechenden Analyse zwar deutlich, jedoch verfehlt er das gewünschte Signifikanzniveau knapp $\left(\mathrm{z}=-1,52 ; \mathrm{p}<0,12\right.$; Koeffizient: $-0,47$; $\mathrm{LR} \mathrm{Chi}^{2}(\mathrm{df}=2)=39,35$; Prob $>\mathrm{Chi}^{2}=0,00$; Pseudo $\mathrm{R}^{2}=0,12$ ). Damit kann Hypothese $4 \mathrm{~b}$ teilweise bestätigt werden.

Offenbar lässt sich die Attraktivität des auf dem ersten Rangplatz präsentierten Fahrzeugs (erklärt durch die Verlustaversion der Individuen) durch die Aktivierung von metakognitiven Vorstellungen nahezu neutralisieren. Personen nutzen Hinweise aus der

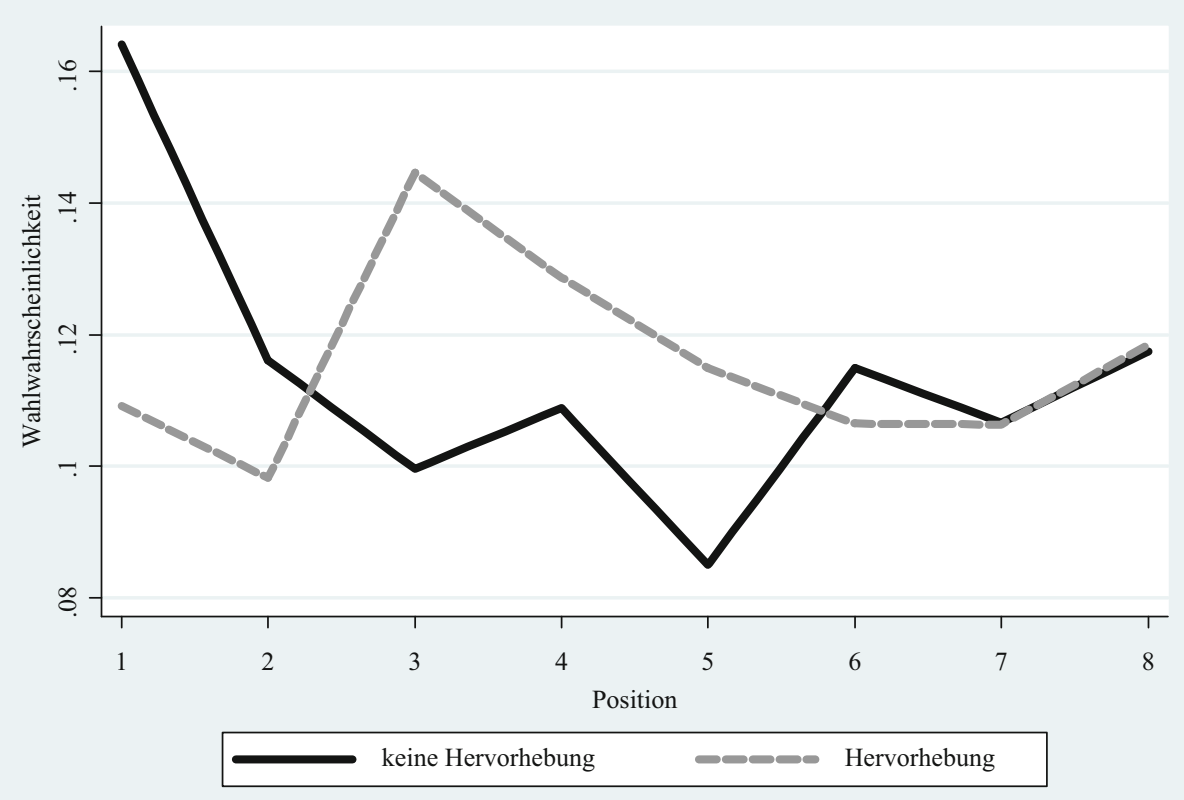

Erläuterung zur Abbildung:

Schwarze Linie = Wahlwahrscheinlichkeit der Bedingung 1 (die Pkw sind in zufälliger Reihenfolge untereinander dargestellt).

Gestrichelte Linie $=\quad$ Wahlwahrscheinlichkeit der Bedingung 5 (die Fahrzeuge sind in zufälliger Reihenfolge untereinander dargeboten, wobei die ersten drei Automobile farblich hervorgehoben sind).

Position $8=$ Konstruierte Referenzposition.

Abb. 5: Auswirkung farbiger Hervorhebungen auf die Wahlwahrscheinlichkeiten 


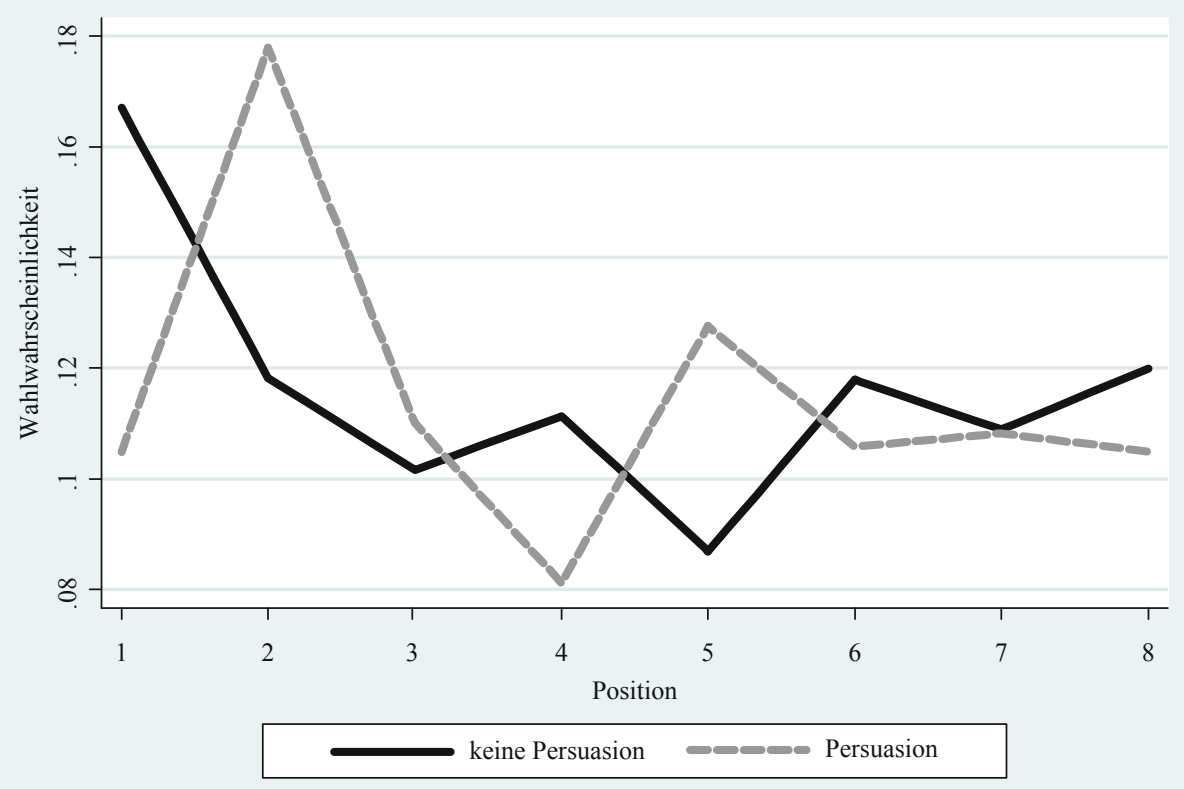

Erläuterung zur Abbildung:

Schwarze Linie = Wahlwahrscheinlichkeit der Bedingung 1 (die Pkw sind in zufälliger Reihenfolge untereinander dargestellt).

Gestrichelte Linie $=$ Wahlwahrscheinlichkeit der Bedingung 6 (die Pkw sind in zufälliger Reihenfolge untereinander angeordnet, und es liegen persuasive Informationen zu den einzelnen Optionen vor).

Position $8=$ Konstruierte Referenzposition.

Abb. 6: Auswirkung persuasiver Information auf die Wahlwahrscheinlichkeiten

Entscheidungssituation, um daraus Regeln über die Art und Weise der Darbietung von Pkw durch den Hersteller abzuleiten. Ist die Vorstellung aktiviert, der Hersteller habe die Absicht, den Entscheider zu beeinflussen, entwickelt sich die Überzeugung, auf dem ersten Platz befinde sich ein für den Kunden unvorteilhaftes Automobil, das schnell und zu einem ungünstigen Preis abgesetzt werden soll.

Anknüpfend daran interessiert, ob Personen in den Konditionen mit Beeinflussungsabsicht (persuasive Informationen bzw. farbige Hervorhebung) eine geringere Entscheidungsqualität aufweisen als in der Referenzgruppe, weil die Pkw-Wahl auf Basis von Vertrauens- oder Misstrauensheuristiken erfolgt (Hypothese 4c). Es sei erinnert, dass es nicht um gute oder schlechte Entscheidungen im objektiven Sinne geht, sondern lediglich um das Ausmaß, in dem die getroffenen Entscheidungen den eigenen Präferenzen besser oder schlechter entsprechen. Eine Regressionsanalyse mit der Entscheidungsqualität als abhängiger Variablen und der Manipulation ,persuasive Informationen“ als unabhängiger Größe liefert einen statistisch signifikanten Effekt $(t=1,97 ; \mathrm{p}<0,05$; Koeffizient: $-0,18)$. Ein zweites Regressionsmodell mit der Manipulation „farbige Hervorhebung“ 
führt zu einem ähnlichen Befund; auch diese Manipulation bewirkt eine Reduktion der Entscheidungsqualität $(t=3,68 ; \mathrm{p}<0,01$; Koeffizient: $-0,33)$. Auf Basis dieses Resultats kann Hypothese 4c als vorläufig bestätigt betrachtet werden.

Nachdem gezeigt werden konnte, dass persuasive Informationen und die farbige Hervorhebung die Qualität der Entscheidungen reduzieren, ist zu prüfen, ob dieser Effekt tatsächlich auf eine Aktivierung metakognitiver Vorstellungen zurückgeführt werden kann (vgl. Hypothese 4d). Wie erläutert liegt die Vermutung nahe, metakognitive Vorstellungen fungieren als Mediator zwischen der persuasiven Information bzw. der farbigen Hervorhebung und den Entscheidungsfehlern.

Ein Sobel-Test bringt zum Ausdruck, dass die metakognitiven Vorstellungen den Effekt der persuasiven Informationen auf die Entscheidungsfehler mediieren (Sobel-TestStatistik: 1,20; $<<0,02)$. Ein ähnlicher Befund zeigt sich auch für die Wirkung der farbigen Hervorhebung; auch hier belegt der Sobel-Test eine Mediation durch die metakognitiven Vorstellungen (Sobel-Test-Statistik: 3,26; p < 0,01). Folglich kann der Zusammenhang zwischen dem Eingriff in die Entscheidungssituation und der Zunahme der Entscheidungsfehler mit den metakognitiven Vorstellungen über die Listenanordnung erklärt werden.

Abschließend ist noch zu prüfen, ob eine Person, die die Überzeugung hegt, die markierten Fahrzeuge seien die besten für sie (Vermutung der positiven Beeinflussung durch den Händler), sich tatsächlich für den hervorgehobenen Pkw entscheidet. Dominiert hingegen die Idee, dass die Automobile markiert sind, die der Anbieter absetzen möchte (negative Beeinflussung), müsste das Individuum zu einem nicht hervorgehobenen Automobil greifen. Einer logistischen Regressionsanalyse zur Folge entfalten die metakognitiven Vorstellungen einer Person eine deutliche Wirkung auf deren Entscheidung. Individuen, die die Vermutung äußerten, die Hervorhebung des Händlers sei auf eine negative Beeinflussungsabsicht zurückzuführen, zeigten eine statistisch signifikant geringere Häufigkeit, ein markiertes Auto auszuwählen $\left(\mathrm{z}=-2,51 ; \mathrm{p}<0,01\right.$; Koeffizient: $-0,99$; LR Chi ${ }^{2}$ $(\mathrm{df}=1)=6,88$; Prob $>\mathrm{Chi}^{2}=0,01$; Pseudo $\left.\mathrm{R}^{2}=0,03\right)$, als jene, die die Autos mit dem besten Preis-Leistungsverhältnis in der Hervorhebung wähnen. Somit ist auch Hypothese $4 \mathrm{e}$ vorläufig bestätigt.

\section{Implikationen für Wissenschaft und Praxis}

Eingangs wurden mit Blick auf die Literatur drei Forschungsfragen aufgeworfen, die auf Basis der erzielten Ergebnisse wie folgt beantwortet werden können: (1) Zunächst ist zu konstatieren, dass sich die Entscheidung einer Person für ein Produkt unabhängig von dessen Beschaffenheit durch seinen Platz in einer Rangreihung beeinflussen lässt. (2) Ferner konnte festgestellt werden, dass Individuen bei randomisierten Listen häufig zur Wahl des ersten Objekts tendieren, während sie in geordneten Listen das mittlere Erzeugnis favorisieren. (3) Darüber hinaus spielen metakognitive Vorstellungen der Nachfrager bezüglich möglicher Kalküle des Anbieters bei der Komposition der Liste eine zentrale Rolle bei der Produktwahl. Die farbliche Hervorhebung von Objekten oder die Präsentation persuasiver Informationen sind Instrumente, um vor der Entscheidung metakognitive Überlegungen zu aktivieren. Vorsicht und Misstrauen prägen in der Folge das Entscheidungsverhalten der Personen, so dass die zuvor (Punkt 2) erwähnten Heuristiken (etwa die Orientierung an dem auf dem ersten Rangplatz präsentierten Objekt) nicht mehr wirksam sind. Diese 
Vorstellungen schlagen sich auch im Verhalten nieder; Personen, die vermuten, das Unternehmen hebt die Produkte mit dem besten Preis-Leistungsverhältnis hervor, neigen eher dazu, diese markierten Erzeugnisse zu wählen, als jene Kunden, die in der Hervorhebung die schlechtesten Objekte wähnen. Hieraus ergeben sich Implikationen für Wissenschaft und Praxis.

\section{- Wissenschaftliche Implikationen}

Zusammenfassend liefern die erzielten Resultate weitere Belege für das Menschenbild des irrationalen Entscheiders. Hier ist insbesondere die Relevanz der „prospect“-Theorie (und die damit verknüpfte Verlustaversion) zu nennen, die die Herausbildung des Vergleichsstandards erklärt. Obgleich die Festlegung eines Ankerpunkts kognitive Kapazitäten erfordert, führt er zu keiner objektiv besseren Entscheidung. Die Alternativhypothesen der Primacy- und Recency-Effekte konnten hingegen im Kontext der Kaufentscheidungen aus Produktlisten widerlegt werden. Auch der Marketplace-Metacognition-Ansatz, bei dem Individuen Überlegungen anstellen, deren Ziel die Ermittlung der Platzierung des attraktivsten Fahrzeuges ist, liefert Bestätigung für den heuristischen Entscheidungsprozess. Dabei liefern die Ergebnisse Bestätigung dafür, dass bestimmte metakognitive Vorstellungen aktiviert werden, sofern entsprechende Hinweisreize in der Entscheidungssituation vorliegen.

Die Untersuchung zeigt zudem die beschränkte Erklärungskraft der „primacy“- und „recency“-Effekte. Zwar konnten diese Ansätze in diversen früheren Studien bestätigt werden, allerdings ging es zumeist nur um zwei Entscheidungsoptionen (Day 1969; Dean 1980; Scarpi 2004). Zudem waren die Expertise und das Involvement der Probanden bezüglich der zu beurteilenden Produkte niedrig. Auch konnte nachgewiesen werden, dass ,primacy“- und „recency“-Effekte nur bei Individuen festzustellen sind, die über die Optionen schlecht informiert sind bzw. nur geringe Präferenzen besitzen (Gierl u. Höser 2002, S. 10 ff.). Diese Befunde lassen den Schluss zu, dass das Entscheidungsverhalten von Personen mit geringer Expertise und niedrigem Involvement von „primacy“- und „recency“-Effekte beeinflusst ist. Dagegen entscheiden Probanden mit hoher Expertise und großem Involvement gemäß der ,prospect"-Theorie.

Ein Blick auf die erhobenen Daten vermag diese Vermutung zu stützen; einerseits sind in der Stichprobe fast ausschließlich Personen mit hoher Expertise und großem Involvement in Bezug auf Autos zu finden (auf der Expertise-Skala wiesen 85\% aller Teilnehmer/innen einen Wert oberhalb des Skalenmittelpunktes auf, bei der Involvement-Skala sogar 95\%). Andererseits führt eine Analyse der Auswahlwahrscheinlichkeit von Platz 1 bei randomisierten Listen zu der Erkenntnis, dass ein hohes „need for cognition“, ein maximierender Entscheidungsstil, eine lange Entscheidungsdauer sowie ein hohes Involvement die Wahrscheinlichkeit für die Wahl des ersten Platzes positiv beeinflussen. Ein möglicher Interaktionseffekt dieser Variablen wäre in späteren Untersuchungen zu bestätigen.

Ein weiterer theoretischer Beitrag dieser Arbeit besteht im Aufzeigen der Bedingungen, unter denen Individuen beim Entscheidungsverhalten den ersten Platz als Referenzpunkt wählen oder sich für einen mittleren Platz als Referenzpunkt entscheiden. Es konnte gezeigt werden, dass in einer komplexen, unstrukturierten Entscheidungssituation, die ein Individuum bei einer randomisierten Liste vorfindet, das zuerst wahrgenommene Objekt als Referenzpunkt bestimmt. Der „compromise effect“ hingegen erfährt Bestätigung bei einer Entscheidung, bei der die Optionen nach einem Kriterium vorsortiert sind. 
- Praktische Implikationen

Anbieter von Produkten oder Dienstleistungen im Internet können aus den diskutierten Studien die Implikation ableiten, Objekte, die besonders häufig verkauft werden sollen, in randomisierten Listen auf dem ersten Listenplatz und in geordneten Listen auf dem mittleren Listenplatz den Nachfragern zu präsentieren. Des Weiteren sollten diese bedenken, dass eine farbige Hervorhebung oder eine persuasive Information zu einer Reduktion der Entscheidungsqualität auf Kundenseite führen kann. In den meisten Situationen sollte ein Unternehmen darauf bedacht sein, eine hohe Entscheidungsqualität zu erzielen, weil zu erwarten ist, dass diese mit diversen betriebswirtschaftlich relevanten Variablen in Verbindung steht, wie zum Beispiel mit geringem Bedauern der Entscheidung, hoher Entscheidungs- und Konsumzufriedenheit sowie starker Loyalität und der Bereitschaft zur Produktempfehlung (Woodruff et al. 1983; Anderson u. Sullivan 1993; Heitmann et al. 2007). Eine farbige Hervorhebung oder die Bereitstellung persuasiver Informationen bieten sich nur dann an, wenn die Kunden Vertrauen in das Unternehmen besitzen. Sofern ein Hersteller oder Händler diese Elemente zur Gestaltung von Produktlisten einsetzen möchte, ist sicherzustellen, dass der Kunde stets die gute Absicht des „Absenders“ vermutet. Entwickelt ein Individuum im Entscheidungsprozess die Vorstellung, der Verkäufer wolle beeinflussen, ist damit zu rechnen, dass es sich Gedanken über jene Rangplätze macht, auf denen das attraktivste bzw. unattraktivste Produkt platziert sein könnte.

Obgleich die Wahl eines Automobils aus einer Online-Liste im Mittelpunkt der Studie stand, liegt der Gedanke nahe, die erzielten Ergebnisse zu generalisieren. Zunächst kommen Online-Shops oder Portale jedweder Art in Betracht, die allesamt unter Rückgriff auf die diskutierten Erkenntnisse gestaltet werden können. Ferner spielen die gewonnenen Einsichten auch in der Offline-Welt eine Rolle. Man denke etwa an Speise- und Getränkekarten in Restaurants, an Werbeprospekte von Supermärkten oder an Verkaufskataloge und -broschüren aller Art; überall präsentieren die Anbieter ihre Erzeugnisse auf Listen.

- Einschränkungen und Forschungsbedarf

Aufgrund von erhebungstechnischen Restriktionen konnte kein vollständig faktorielles Design verwendet werden. Somit bietet sich in weiteren Studien eine Untersuchung der Auswirkungen der Manipulationen ,persuasive Information“ und ,farbige Hervorhebung“ bei geordneten sowie nebeneinander angeordneten Listen an. Diese Beschränkung betrifft auch die Anzahl der in einer Liste dargebotenen Produkte. Im Sinne eines tieferen Verständnisses der interessierenden Effekte sollten weitere Experimente mit einer variierenden Anzahl von Objekten durchgeführt werden.

Weiterhin sind die Ergebnisse mit Vorsicht zu interpretieren, weil die Beliebtheitskontrollvariable, die zur Identifikation des Positionseinflusses unerlässlich ist, eine sehr starke Variation aufweist. So schwanken die aggregierten Wahlhäufigkeiten der verschiedenen Autos zwischen 0,79 und 46,62\%.

Darüber hinaus müsste die Aufmerksamkeit auch der Kausalitätsproblematik bei der Interpretation der Wirkung metakognitiver Vorstellungen gelten. Da diese Vorstellungen nur abgefragt, nicht jedoch manipuliert wurden, besteht die Gefahr, dass die Nennungen über den Grund der farbigen Hervorhebung nur der Rechtfertigung der bereits getroffenen Entscheidung dienen (Festinger 1957). Dieser Einwand ließe sich durch eine experimentelle Replikation der Untersuchung widerlegen, wobei gezielt die Vertrauenswürdigkeit des Herstellers oder Händlers zu manipulieren wäre. 
Des Weiteren zeigt die Analyse auch bei randomisierten Listen, in denen die Erzeugnisse nebeneinander präsentiert sind, einen deutlichen Ausschlag der Kaufbereitschaft für das in der Mitte angeordnete Produkt. Der Grund für dieses Interesse am mittleren Objekt lässt sich nicht klar und eindeutig aus theoretischen Überlegungen ableiten, so dass weitere Forschung unerlässlich erscheint.

Abschließend haben Analysen der Unterschiede zwischen untereinander und nebeneinander angeordneten Listen ergeben, dass bei untereinander angeordneten Listen das Produkt auf Position 6 signifikant häufiger gewählt wird als bei nebeneinander angeordneten. Dieser Effekt zeigte sich sowohl bei randomisierten als auch bei geordneten Listen. Einer Erklärung dieses Unterschiedes wäre in weiteren Studien nachzugehen.

Danksagung: Wir danken zwei anonymen Gutachtern für wertvolle Hinweise.

\section{Anmerkungen}

1 Unter einer „Rangliste“ wird im Folgenden eine Liste verstanden, in der Produkte oder Unternehmen optisch präsentiert werden. Die Anordnung erfolgt entweder randomisiert oder nach einem beliebigen Sortierkriterium. Als solches Kriterium kann zum Beispiel der Preis oder ein spezifisches Produktmerkmal fungieren. Die verschiedenen Optionen können entweder untereinander oder nebeneinander präsentiert werden.

2 Im Rahmen von Vorarbeiten zu diesem Aufsatz fanden Gespräche mit Vertretern von OnlineShops sowie Auktions- und Buchungsportalen statt. Ohne den Anspruch auf Generalisierbarkeit zu erheben, lässt sich konstatieren, dass bei der Gestaltung von Produkt- oder Trefferlisten Überlegungen über das Entscheidungsverhalten der Individuen eine Rolle spielen. Zumeist liegen den Unternehmen keine Marktforschungserkenntnisse vor, jedoch entwickeln Marktforscher, Produktmanager etc. Szenarien über das Verhalten der Kunden bei der Wahl eines Objekts aus einer Liste. Auf Basis dieser Szenarien erarbeiten die zuständigen Manager Vorgaben für die Gestaltung von Listen.

3 Dies lässt sich anhand von Literaturrecherchen nachvollziehen, indem man zum Beispiel im Web of Science nach den Titelstichworten ,presentation order“, „order effects“, „,rank order“ oder „Reihenfolgeeffekte“ kombiniert mit den Begriffen „choice“, „preference“, „,decision“, „Wahl“, „Entscheidung“ oder „Präferenz“ recherchiert.

4 Aufgrund einer möglichen Benachteiligung einzelner Autoverkäufer war es nicht möglich, die realen Verkaufslisten der Gebrauchtwagenbörse zu manipulieren. Deshalb wurde zur Untersuchung des Themas eine Liste so nachgestellt, dass sie einer realen Verkaufsliste, nach Vordefinition bestimmter Suchkriterien, nahe kommt. Insofern können in der Studie nur hypothetische, nicht aber reale Entscheidungen erfasst werden. Da es Belege für eine beachtliche Deckungsgleichheit zwischen hypothetischen und realen Entscheidungen gibt (Kühberger 2002), soll die Entscheidung als abhängige Variable fungieren, wohl wissend, dass es sich dabei nicht um realisierte, sondern um hypothetische Entscheidungen handelt. Es ist dennoch zu vermuten, dass eine hypothetische Entscheidungssituation mehr Aufschluss über das tatsächliche Entscheidungsverhalten liefert als eine Abfrage der Präferenzurteile aller präsentierten Produkte auf einer Likert-Skala (z. B. bei Eisenberg u. Barry 1988; Dean 1980; Scarpi 2004), weil eine mehrstufige Likert-Skala sensitiv auf kleinste Veränderungen in den Präferenzen reagiert; eine Überprüfung, inwieweit solche minimalen Präferenzunterschiede tatsächlich entscheidungsrelevant sind, ist nur anhand einer dichotomen Entscheidungssimulation zwischen den einzelnen Alternativen möglich. 
5 Es muss darauf hingewiesen werden, dass es sich bei der ,tatsächlich getroffenen“ Entscheidung um eine hypothetische Wahl handelt. Bei der Erfassung dieser Variable ist mit Abweichungen von der realen Entscheidung zu rechnen. Dennoch gehen wir davon aus, dass dies die beste Möglichkeit zur Operationalisierung der ,realen Entscheidung“ ist und dass mögliche Beeinflussungsmechanismen in dieser Situation ähnlich wie in einer realen Entscheidung wirken sollten. Die Präferenznennungen auf den drei einzelnen Rating-Skalen hingegen sind als unbeeinflusste Standardmessung der individuellen Präferenzen zu betrachten, weil es sich dabei um ein Maß handelt, das in allen Konditionen auf die gleiche Art erhoben wurde und von entsprechenden Manipulationen unbeeinflusst blieb. Im Kern zielt die Messung der Entscheidungsqualität darauf ab, zu erfassen, ob die Versuchsteilnehmer in der Entscheidungssituation trotz wahrgenommener Beeinflussungsabsicht an den Attributspräferenzen festhalten, die sie in einer davon unabhängigen Präferenzmessung geäußert haben.

6 Unter der abhängigen Variable „Entscheidung“ soll hier die Auswahl eines spezifischen Fahrzeugs verstanden werden. Diese wird in Abhängigkeit von den dummy-kodierten Listenplätzen untersucht.

\section{Literatur}

Abdellaoui A, Vossmann F, Weber M (2005) Choice-based elicitation and decomposition of decision weights for gains and losses under uncertainty. Manag Sci 51:1384-1399

Anderson EW, Sullivan MW (1993) The antecedents and consequences of customer satisfaction for firms. Marketing Sci 12(2):125-143

Baron RM, Kenny DA (1986) The moderator-mediator variable distinction in social psychological research: conceptual, strategic, and statistical considerations. J Pers Soc Psychol 51(16):1173-1182

Brown CL, Krishna A (2004) The skeptical shopper: a metacognitive account for the effects of default options on choice. J Cons Res 31:529-539

Campbell MC, Kirmani A (2000) Consumers' use of persuasion knowledge: the effects of accessibility and cognitive capacity on perceptions of an influence agent. J Cons Res 27:96-83

Chernev A (2004) Extremeness aversion and attribute-balance effects in choice. J Cons Res 31:249263

Darke PR, Ritchie RJB (2007) The defensive consumer: advertising deception, defensive processing, and distrust. J Marketing Res 44:114-127

Day RL (1969) Position bias in paired product tests. J Marketing Res 6:98-100

Dean ML (1980) Presentation order effects in product taste tests. J Psychol 105:107-110

Deese J, Kaufman RA (1957) Serial effects in recall of unorganized and sequentially organized verbal material. J Exp Psychol 54:180-187

Diehl K, Zauberman G (2005) Searching ordered sets: evaluations from sequences under search. J Cons Res 31:824-832

Eisenberg M, Barry C (1988) Order effects: a study of the possible influence of presentation order on user judgments of document relevance. J Am Soc Inf Sci 39:293-300

Festinger L (1957) A theory of cognitive dissonance. Stanford University Press, Stanford

Friestad M, Wright P (1994) The persuasion knowledge model: how people cope with persuasion attempts. J Cons Res 21:1-31

Friestad M, Wright P (1995) Persuasion knowledge: lay people's and researchers' beliefs about the psychology of advertising. J Cons Res 22:62-74

Friestad M, Wright P (1999) Everyday persuasion knowledge. Psychol Mark 16:185-194

Gierl H, Höser H (2002) Der Reihenfolgeeffekt auf Präferenzen. Z betriebswirtschaftliche Forsch $54: 3-18$ 
Glanzer M, Cunitz AR (1966) Two storage mechanisms in free recall. J Verbal Learn Verbal Behav 5:351-360

Heitmann M, Lehmann DR, Herrmann A (2007) Choice goal attainment and decision and consumption satisfaction. J Marketing Res 44:234-250

Jain SP, Posavac SS (2001) Prepurchase attribute verifiability, source credibility, and persuasion. J Consum Psychol 11:169-180

Johnson EJ, Payne JW (1985) Effort and accuracy in choice. Manag Sci 31:394-414

Kahneman D, Frederick S (2002) Representativeness revisited: attribute substitution in intuitive judgment. In: Gilovich T, Griffin D, Kahneman D (Hrsg) Heuristics and biases: the psychology of intuitive judgment. Cambridge University Press, Cambridge, S 49-81

Kahneman D, Tversky A (1979) Prospect theory: an analysis of decision under risk. Econometrica 47:263-291

Kardes FR, Kalyanaram G (1992) Order-of-entry effects on consumer memory and judgment: an information integration perspective. J Marketing Res 29:343-357

Kardes FR, Sanbonmatsu DM (1993) Direction of comparison, expected feature correlation, and the set-size effect in preference judgment. J Consum Psychol 2(1):39-54

Kivetz R, Netzer O, Srinivasan V (2004) The extending compromise effect models to complex buying situations and other context effects. J Marketing Res 23:237-257

Kühberger A, Schulte-Mecklenbeck M, Perner J (2002) Framing decisions: hypothetical and real. Organ Behav Hum Decis Process 89:1162-1175

Murdock BB Jr (1962) The serial position effect of free recall. J Exp Psychol 64(5):482-488

Murphy J, Hofacker C, Mizerski R (2006) Primacy and recency effects on clicking behavior. J Comput-Mediat Commun 11:522-535

Mussweiler T (2003) Comparison processes in social judgment: mechanisms and consequences. Psychol Rev 110(3):472-489

Novemsky N, Kahneman D (2005a) The boundaries of loss aversion. J Marketing Res 119:119-128

Novemsky N, Kahneman D (2005b) How do intentions affect loss aversion? J Marketing Res 42:119 128

Pan B, Hembrooke H, Joachims T (2007) In google we trust: users' decisions on rank, position, and relevance. J Comput-Mediat Commun 12:801-823

Payne JW, Bettman JR, Johnson E (1993) The adaptive decision maker. Cambridge University, Cambridge

Piller FT (2004) Mass customization: reflections on the state of the concept. Int J Flex Manuf Syst $16: 313-334$

Polak B, Herrmann A, Heitmann M, Einhorn M (2008) Die Macht des Defaults - Wirkung von Empfehlungen und Vorgaben auf das individuelle Entscheidungsverhalten. Z Betriebswirtschaft 78:1033-1060

Prelec D, Wernerfelt B, Zettelmeyer F (1997) The role of inference in context effects: inferring what you want from what is available. J Cons Res 24:118-125

Raanaas RK, Magnussen S (2006) Serial position effects in implicit memory. Eur J Cogn Psychol 18:398-414

Samuelson W, Zeckhauser R (1988) Status quo bias in decision making. J Risk Uncertainty 1:17-59

Scarpi D (2004) Effects of presentation order on product evaluation: an empirical analysis. Int Rev Retail Distrib Consum Res 14:309-319

Schweitzer M (1994) Disentangling status quo and omission effects: an experimental analysis. Organ Behav Hum Decis Process 58:457-476

Shami NS, Ehrlich K, Millen D (2008) Pick me! Link selection in expertise search results. In: SIGCHI conference on human factors in computing system, Florence, Italy, 10 April $2008 \mathrm{~S}$ 1089-1092

Sheng S, Parker AM, Nakamoto K (2005) Understanding the mechanism and determinants of compromise effects. Psychol Mark 22:591-609 
Simonson I (1989) Choice based on reasons: the case of attraction and compromise effects. J Cons Res 16:158-174

Simonson I, Tversky A (1992) Choice in context: tradeoff contrast and extremeness aversion. J Marketing Res 29:281-295.

Terry WS (2005) Serial position effects in recall of television commercials. J Gen Psychol 132:151163

Thaler RH (1985) Mental accounting and consumer choice. Marketing Sci 4:199-214

Thaler RH, Johnson EJ (1990) Gambling with the house money and trying to break even: the effects of prior outcomes on risky choice. Manag Sci 36:643-660

Tversky A, Kahneman D (1991) Loss aversion in riskless choice: a reference-dependent model. Quart J Econ 106:1039-1061

Wernerfelt B (1995) A rational reconstruction of the compromise effect: using market data to infer utilities. J Cons Res 21(4):627-633

Woodruff RB, Cadotte ER, Jenkins RL (1983) Modeling consumer satisfaction processes using experience-based norms. J Marketing Res 20(3):296-304

Wright P (2002) Marketplace metacognition and social intelligence. J Cons Res 28:677-682

\title{
Rank order matters-Effect of rank order of products on individual decision making
}

\begin{abstract}
Customers often search for and choose from ordered sets. E. g., online shops present their product assortments as lists; very often the products are listed according to criteria as defined by the customers. Based on theoretical explanations it can be assumed that the rank of a product in a list effects its demand. An empirical study run in the market for used cars shows the importance of that effect. The study also reveals the most preferred rank and provides further insights for an optimal ordering of products. Furthermore, the understanding of individual decision about rank ordered products can be deepened.
\end{abstract}

Keywords: Product order ' Rank order - Ranking $\cdot$ Compromise effect $\cdot$ Marketplace metacognition $\cdot$ Prospect theory $\cdot$ Recency and primacy $\cdot$ Experimental design 